Article

\title{
Impact of Road Geometry on Vehicle Energy Consumption and $\mathrm{CO}_{2}$ Emissions: An Energy-Efficiency Rating Methodology
}

\author{
Hugo Ferreira ${ }^{1}$, Carlos Manuel Rodrigues ${ }^{2}$ and Carlos Pinho ${ }^{3, *(1)}$ \\ 1 Department of Mechanical Engineering, School of Technology and Management, Polytechnic of Viseu, \\ Campus Politécnico, s/n, 3504-510 Viseu, Portugal; hugomoreira@ipv.pt \\ 2 CITTA, Faculty of Engineering, University of Porto, Rua Dr. Roberto Frias, s/n, 4200-465 Porto, Portugal; \\ cmr@fe.up.pt \\ 3 CEFT, Faculty of Engineering, University of Porto, Rua Dr. Roberto Frias, s/n, 4200-465 Porto, Portugal \\ * Correspondence: ctp@fe.up.pt
}

Received: 5 November 2019; Accepted: 22 December 2019; Published: 25 December 2019

\begin{abstract}
This study presents a methodology for classifying road traffic energy efficiency. The indicators defined discriminate the impact of the road vertical and horizontal alignments upon energy consumption, disclosing the improvement potential of the road as a function of the traffic origin-destination matrix. The methodologic approach is based on basic physical principals, thus guarantying its generality, as opposed to the usual empirical mesoscale approaches. A simplified algebraic procedure is also proposed, resorting to simplified driving cycles and a constant speed assumption (CSA), thus avoiding the intricacy of microscale/microsimulation models. The simplified methodology was validated against field data acquired on the Portuguese highway A25. A microscale vehicle specific power analysis combined with detailed fuel models is compared against CSA results. The findings demonstrate its adequacy for free-flow traffic conditions and the importance of classifying road traffic energy-efficiency. For the case studied, it was found that $49.5 \%$ of the round trip propulsive energy expended by a 37-ton truck on the A25, a modern road, was degraded as heat through braking. The difference found between the microscale analysis and CSA approach is $0.8 \%$, despite the speed unevenness, varying between 32 and $96 \mathrm{~km} / \mathrm{h}$, with a standard deviation of $24 \%$ of the average speed.
\end{abstract}

Keywords: traffic energy-efficiency; road geometry; fuel consumption; $\mathrm{CO}_{2}$ emissions; free-flow driving cycles

\section{Introduction}

The need to expand the knowledge on road energy consumption in a variety of scenarios, reflecting road geometry, vehicle characteristics, and control strategies, is more pertinent today than ever before. Numerous studies have addressed the question of quantifying the energy demand and emissions exhibited by different types of vehicles, in order to develop fuel consumption and emissions models [1-7], to adjust driving cycles to real-world conditions [8-15], to determine the energy-efficiency of electrical vehicles (EV) or the benefits of regenerative braking in a hybrid powertrain [16-19], and to adopt eco-friendly driving strategies through vehicle control optimization [20-22] or the practice of eco-routing [23-26].

However, the question of quantifying the motion efficiency through a bounded dimensionless indicator $0 \leq \eta \leq 1$ capable of establishing the margin of improvement of a road alignment rests unaddressed, although the path and the motion that minimizes propulsive work is well known: a geodesic straight line traversed at a constant speed $[27,28]$. The difficulty lies in quantifying how 
close the real-world energy consumption is from the ideal scenario through an algebraic comprehensive calculus procedure. This can only be done for a given gradient and speed level $[5,29]$. For real driving cycles, the estimation of propulsive work, fuel efficiency, and emissions demands the integration of field data based on the vehicle specific power (VSP) [30-32], or the processing of computationally generated speed profiles over a given route. The latter approach is possible using microscopic models, such as those embedded in traffic simulators like SUMO [33] or VISSIM [34], and then computing fuel efficiency or emissions through microsimulation models like PHEM [35], CMEM [36], or VT-Micro [5]. However, these microscale computational models are opaque to the user.

All of the above mentioned studies, and those discussed on the literature review section, are focused on illustrating the road grade impact upon energy consumption, or emissions, based on the analysis of particular routes, empirically or through microanalysis, rather than establishing cause-effect relationships through physical principles only, without which any energy-efficiency rating procedure loses generality. The question of quantifying energy consumption through a comprehensive and dimensionally homogeneous model, defined as a function of significant vehicle parameters and road geometry, is the main objective of the present study. In this regard, an energy-efficiency rating procedure is presented, based on the algebraic modelling of the road traffic energy consumption and valid only for free-flow conditions. This limitation allowed the use of constant speed driving cycles and was validated against field data obtained on the Portuguese A25, a hilly motorway, on a second-by-second basis, by studying different driving patterns. The motion efficiencies of conventional passenger cars and heavy-duty trucks (HDT) are examined. The effect of the road geometry upon the energy performance of the vehicles is evaluated through detailed energy balances, discriminating the mechanical energy change, the rolling and drag resistances, and the energy losses ensuing from braking and powertrain friction. Finally, a hypothetical traffic scenario is analyzed in order to illustrate the physical meaning of the efficiency indicators. The results show that the fuel consumption and greenhouse gas (GHG) emissions of heavy-duty vehicles are largely affected by road geometry, stressing the importance of an energy-efficiency rating procedure.

\section{Literature Review}

\subsection{Road Energy-Efficiency Classification}

The pertinence of an efficiency indicator correlating traffic energy demand and road alignment was recognized recently by Luin et al. [28]. These authors addressed the question and proposed the Energy Loss Index (ELI). It is defined as the ratio between the energy consumed on the road $E_{\text {road }}$ and the energy that would be needed on an ideal path $E_{\text {ideal }}$, naturally for the same traffic, divided in $c$ classes each composed by a given number of vehicles $n_{c}$ and exhibiting a class energy demand $E_{c}$, computed through the VSP methodology [28].

$$
\begin{gathered}
E L I=\frac{E_{\text {road }}}{E_{\text {ideal }}} \\
E_{\text {road }}=\sum_{c=1}^{N} n_{c} E_{c} \\
E_{c}=m_{\mathcal{c}} \int_{0}^{T} \dot{w}_{P} d t
\end{gathered}
$$

The propulsive vehicle specific power $\dot{w}_{P}$ considers four components (Equation (4)): $\dot{w}_{G E}$, the gravitational energy rate of change, determined by the road slope $s ; \dot{w}_{K E}$, the kinetic energy rate of change, dependent on the acceleration $a ; \dot{w}_{R R}$, the tires rolling resistance specific power, dependent on the rolling resistance coefficient $C_{R R}$; and the aerodynamic drag component $\dot{w}_{D}$, proportional to the vehicle frontal area $A$, drag coefficient $C_{D 0}$, and air density $\rho_{a}$. The procedure also contemplates the effect of a longitudinal natural wind, with velocity $v_{w}$, and the inertia of the vehicle rotating 
components, through the dimensionless mass factor $\epsilon$ : equal to the rotational kinetic energy of the wheels, transmission and engine, divided by the vehicle translational kinetic energy [30,37].

$$
\begin{gathered}
\dot{w}_{P}=\dot{w}_{G E}+\dot{w}_{K E}+\dot{w}_{R R}+\dot{w}_{D} \\
=g s v+(1+\epsilon) a v+g C_{R R}(v) v+\frac{\rho_{a} C_{D 0} A}{2 m}\left(v+v_{w}\right)^{2} v
\end{gathered}
$$

When the wind speed time-series is unknown, $\dot{w}_{D}$ is obtained from the weighted wind drag coefficient $C_{D W}$ [38],

$$
\dot{w}_{D}=\frac{F_{D} v}{m}=\frac{\rho_{a} C_{D W} A}{2 m} v^{3} .
$$

The ELI methodology presents several limitations: (i) it assumes the ideal solution as a single path, straight between consecutive road nodes; (ii) it does not discriminate the mechanical energy component from the total propulsive energy; (iii) it is not actually bounded, ELI $\in]-\infty,+\infty[$, impairing its usefulness for road comparison purposes; and (iv) it distorts the impact of the gradient profile upon the vehicle energy consumption, by sanctioning the use of a single conservative vehicle specific power model and by not bounding it to a positive quantity.

Regarding point (i), it is imperative to stress that a road provides access to several significant areas from the point of view of human activity. Therefore, its curving nature can actually express a service and not simply a more or less efficient constructive solution. As such, straightening the path between consecutive nodes may not produce a very different solution from the one actually implemented. In this study, the authors propose an ideal solution in which the road links are independent from each other, i.e., the interest of a given user does not constrain that of another, and the energy consumption is minimized. The solution is a non-planar network [39] composed by geodesic straight lines directly connecting the road nodes, Figure 1. Although utopic, it constitutes a relevant design goal and a reference to establish the margin of improvement of the road.

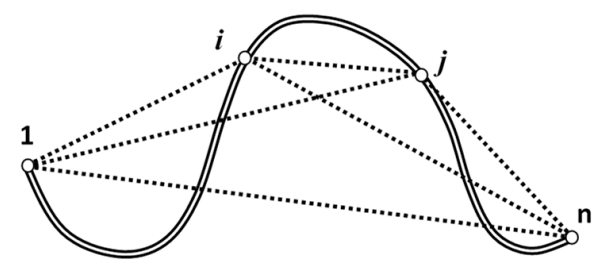

Figure 1. Ideal non-planar road network.

Regarding point (ii), the ELI concept is intended as an energy loss index; however, the vehicle mechanical energy is not an energy loss, being available for propulsion beyond the road exiting point. Additionally, for road comparison purposes, the inclusion of the gravitational energy component is indifferent, since it is independent from the path followed. Moreover, the mechanical energy considered in both terms of the ELI concept can result in zero or negative values of ideal propulsive energy, namely while travelling on an ideal straight route with a steep negative grade, while on the real road this quantity could still be positive or not as negative. These circumstances can produce a positive ELI value lower than 1 (when both terms are negative) or tend to $-\infty$ or $+\infty$ (for near zero values of ideal energy consumption), thus undermining its usefulness as an efficiency index, limitation (iii). Regarding point (iv), in the present paper, the authors defend that propulsive energy should be expressed explicitly as a function of the specific motion resistance (per unit of weight), rather than solely through VSP modelling, since the gradient profile severity and its impact upon energy losses through braking cannot be decoupled from the specific motion resistance force $f_{S M R}$. This dimensionless quantity can be obtained from Equation (4) for a constant mechanical energy,

$$
f_{S M R}=\frac{\dot{w}_{P}}{g v}, a=0 \wedge s=0
$$


In fact, by applying to all vehicle classes a VSP model of a light-duty vehicle (LDV), Luin et al. [28] endorse a value of $15.7 \mathrm{~W} / \mathrm{kg}$ for a heavy-duty truck travelling at $105 \mathrm{~km} / \mathrm{h}$. This speed is typical of a class 8 HDTs in USA, opposed to the implied cruising power of $565 \mathrm{~kW}$ for a 36 metric tons truck [40], the equivalent to a specific motion resistance of $5.5 \%$ of the vehicle weight $\left(f_{S M R}=0.055\right)$. This also implies that travelling at this speed over a flat road or over a hilly one, with grades up to $\pm 5.5 \%$, would require the same propulsive energy since mechanical energy degradation through braking would not occur in either case. This distorts the gradient profile impact upon energy consumption. This distortion is further aggravated by an unbounded VSP model (to positive values) while computing the class propulsive energy $E_{c}$ that determines the fuel consumption $V_{F C}$, ensuing from the engine average thermal efficiency $\eta_{t h}$ and from the fuel specific heat content $H_{f}$, converted to a volume basis by considering its density $\rho_{f}$, as in Luin et al. [28],

$$
V_{F C}=\frac{E_{c}}{\rho_{f} H_{f} \eta_{t h}} .
$$

An internal combustion engine is not a reversible heat engine [41]; it does not store energy. Its fuel consumption is a monotonically increasing quantity with time $t$, being strongly correlated with the positive propulsive power component only [42], $\dot{w}_{P}$ (Equation (4)). In addition, the integration of the grade dependent term in the VSP model (Equation (4)) simply reflects the change in potential energy between the boundary points since $\int s v d t=\int s d x=\Delta z$. Therefore, an unbounded integration of $\dot{w}_{P}$ does not capture the dissipation of mechanical energy through braking on the steepest downgrades, regardless of its magnitude. Similarly, the integration of the inertial dependent term equals the variation of kinetic energy between boundary points, $\int v a d t=\int v d v=\Delta v^{2} / 2$, thus failing to depict the effect of any acceleration-deceleration cycle on the road. As a result, the fuel model advanced by Luin et al. [28] (Equation (7)), and embedded in the ELI analysis, is limited to runs that satisfy the very restrictive condition $\dot{w}_{P}(t) \geq 0$. The fuel models, and related emissions, demand a more detailed analysis and a bounded VSP analysis. In the present study, this path is followed and actual known vehicle specifications are used, being the fuel consumption obtained through high-level fuel models $[1,43]$, torque and speed based.

\subsection{Fuel Models}

The fuel consumption of the test car is computed through the model of Ahn [44], being the fuel rate per unit of engine rotation $\dot{m}_{S F} / \omega$ defined by polynomial functions of the torque $\tau$ and engine speed $\omega$,

$$
\frac{\dot{m}_{S F}(\omega, \tau)}{\omega}=\sum_{i=0}^{2}\left(\sum_{j=0}^{2} c_{i, j} \omega^{j}\right) \tau^{i}
$$

The engine torque in the $n_{\text {th }}$ gear is computed from the instantaneous engine power $\dot{W}_{E}$ ensuing from the road VSP load $\dot{w}_{P}$, and the transmission mechanical efficiency $\eta_{t r}$. The engine speed is obtained from the wheel radius and overall transmission gear ratios.

$$
\tau=\frac{\dot{W}_{E}}{\omega}=\frac{m \dot{w}_{P}(v) / \eta_{t r}}{\omega(v, n)}
$$

For vehicles equipped with gasoline engines, this model is applied to stoichiometric operation only. For higher engine loads, the activation of protective measures to prevent knock, namely, mixture enrichment and ignition retarding, determine a strong increase in specific fuel consumption. For this reason, a corrective function was applied to Equation (8), in the form of a power law of the torque in excess of the upper stoichiometric boundary [45], and the model applied to the EPA engine fuel map [46]. Figure 2 shows the computed thermal efficiency map and the parity line between modeled and measured efficiencies [46]. 
Regarding diesel engines, the CMEM model (comprehensive modal emissions model) was used [47]. The model inputs are: the engine displacement $D$; the friction factor $K$, expressed as a idling energy consumption per unit volume (of $D$ ); the engine indicated thermal efficiency $\eta_{\text {ind }}$; and a reference engine speed $\omega_{0}$.

$$
\begin{gathered}
\dot{m}_{S F} \times H_{f}=\left(K D \omega+\frac{\dot{W}_{E}}{\eta_{\text {ind }}}\right)\left[1+\alpha \times\left(\omega-\omega_{0}\right)^{2}\right] \\
K=K_{0} \times\left[1+\beta \times\left(\omega-\omega_{0}\right)\right] \\
H_{f}=42.9 \mathrm{MJ} / \mathrm{kg}, \quad K_{0}=31.8 \mathrm{~J} / \mathrm{dm}^{3}, \quad \alpha=(2 \pi)^{-2} \times 10^{-4}, \quad \beta=0.00125 / 2 \pi
\end{gathered}
$$

The coefficients $\alpha$ and $\beta$ are proposed by Barth et al. [47], and the $K_{0}$ value complies with the idling fuel rate advanced by Rakha et al. [31]. Under wide-open throttle operation, the quantity $\omega_{0}$ nearly coincides with the most economical engine speed. Therefore, for the heavy-duty trucks studied, this parameter and $\eta_{\text {ind }}$ were inferred from the fuel map considered in EPA and NHTSA [48] for the 2018 baseline class 8 truck. For diesel passenger cars, an optimum brake thermal efficiency of $40 \%$ is assumed at half the rated engine speed.

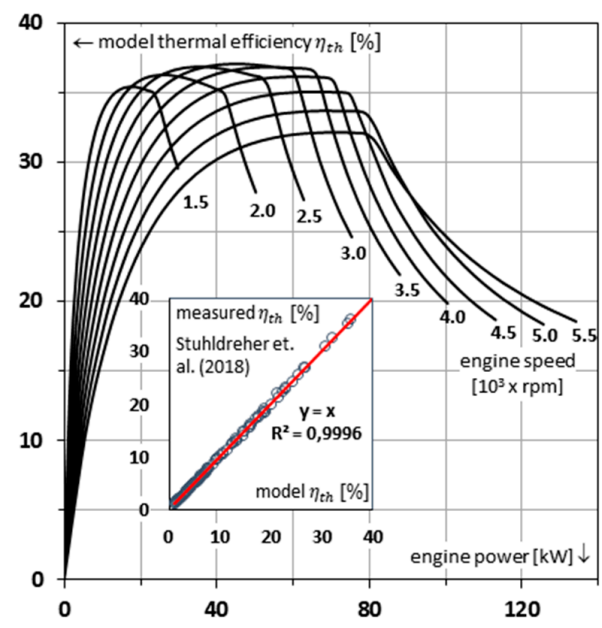

Figure 2. Brake thermal efficiency map of the test car and its parity with measured values, red line.

\subsection{Energy Consumption vs. Road Grades}

The road grade influence upon the fuel consumption (FC) of a medium size car was studied by Boriboonsomsin and Barth [29], through field measurements while travelling at $96 \mathrm{~km} / \mathrm{h}$ over alternative routes, one flat and the other hilly. Additionally, the authors combine a VSP analysis with a CMEM fuel model to obtain unbiased results as a function of speed and grade, ranging from $-8 \%$ to $8 \%$. The field results suggest that the hilly road, with a $6 \%$ ruling grade, increased FC by as much as $20 \%$ when compared to the flat road. In Frey et al. [49], the effect of grade and route choice upon the fuel use of several light-duty vehicles was also studied based on field measurements. The authors conclude that the gradient profile has a considerable impact on fuel use on a microscale, at the segment level, with slopes ranging from $-8 \%$ to $8 \%$, opposed to what happens on a mesoscale, for the entire trip. For the latter, the findings point to an FC impact up to 3.2\% when road grades are disregarded in a VSP modal analysis [30]. These somewhat conflicting findings stress the importance of road characterization and VSP analysis against particular field measurements, in order to properly isolate the contributing factors to FC. Wind or platooning effects were not addressed. Regarding heavy-duty trucks HDTs, the Oak Ridge Laboratory carried out a large scale field study on the effect of weight, road grade, and average speed upon the fuel efficiency of Class- 8 trucks [40]. It was concluded that the fuel consumption is strongly dependent on weight and type of terrain. In Levin et al. [50], the impact of road grade on the network energy consumption of two cities is studied. The authors resort to the rate of change of the average traffic speeds in order to estimate the energy consumption through a positive propulsive power model. The results 
show that road grade should not be disregarded in any eco-routing analysis. Similar findings were reached by Wyatt et al. [42], or in the large-scale field study of Sentoff et al. [32]. In the latter, 700 hours of real-world data were processed through a VSP modal analysis and MOVES2010 (Motor Vehicle Emissions Simulator) [51], the results being categorized by vehicle activity (VSP mode) and road scenario (urban or rural). It was concluded that disregarding road grade underestimates, the energy consumption went from $10 \%$ to $20 \%$. Other authors have addressed the same subject resorting to different explanatory variables, such as Gallus et al. [15], who correlated road grade and cumulated altitude gain with emissions measurements obtained through PEMS (portable emission measurement system). However, the gradient profiles were not typified nor its effect studied through comprehensive approaches.

\section{Definitions}

\subsection{Propulsive and Vertical Alignment Efficiencies}

The propulsive efficiency of a conventional vehicle, $\eta_{P} \in[0,1]$, Equation (11), is defined as the ratio between the minimum unavoidable propulsive energy losses intrinsic to vehicle motion, $E_{L, \text { motion }}$ and the total energy losses experienced on the road, $E_{L, \text { road }}$. This is equivalent to the ratio between the propulsive level-road work $W_{\bar{v}}$, Equation (12), at the constant speed $\bar{v}$ [27], and the corrected positive propulsive work $W_{C P}$ expended on the road, Equation (13), with the slope distribution $s$ and length $L$, for the same travel time $t_{T}=\int v^{-1} d x=L / \bar{v}$. For constant speed driving cycles, the propulsive energy losses ensues form vehicle motion, due to the aerodynamic drag $F_{D}$ and the rolling resistance $F_{R R}$, and from sustained braking on the steepest downgrades. The propulsive efficiency in such cases is denoted as vertical alignment propulsive efficiency $\eta_{V A P}$ :

$$
\begin{gathered}
\left.\eta_{V A P}=\eta_{P}\right]_{v=\bar{v}}, \quad \eta_{P}=\frac{E_{L, \text { motion }}}{E_{L, \text { road }}}=\frac{W_{\bar{v}}(v)}{W_{C P}(s, v)}, \\
W_{\bar{v}}=\left(F_{R R}+F_{D}\right)_{v=\bar{v}} \times L, \\
W_{C P}=W_{P}^{+}-\Delta E_{M}=m \int_{0}^{t_{T}} \dot{w}_{P}^{+} d t-\Delta E_{M} .
\end{gathered}
$$

The corrected work does not reflect the mechanical energy change $\Delta E_{M}$ between boundary points, since this is not an energy loss. The positive propulsive work $W_{P}^{+}$is obtained through a VSP analysis bounded to positive values, $\dot{w}_{P}^{+}$. The use of the positive propulsive work $W_{P}^{+}$is imperative since an ICE engine is not a reversible heat engine. This quantity can be obtained from an energy balance, Equation (14), considering for simplicity reasons the vehicle as non-motored, and the positive propulsive force $F_{P}^{+}$as an external action applied to its center of mass, Figure 3. This action is the only positive energy transfer to the vehicle body, responsible for overcoming its inherent resistance $F_{R}$ and for balancing the mechanical energy, kinetic and potential, dissipated during braking $W_{B}=\int F_{B} d x$ and stored in the vehicle $\Delta E_{M}$. The work components in Equation (15) are always positive as they only reflect the action of dissipative forces, thus bounding $\eta_{V A P}$ to a positive quantity that tends to one when the energy dissipation is imputable to the vehicle only and not to the road alignment.

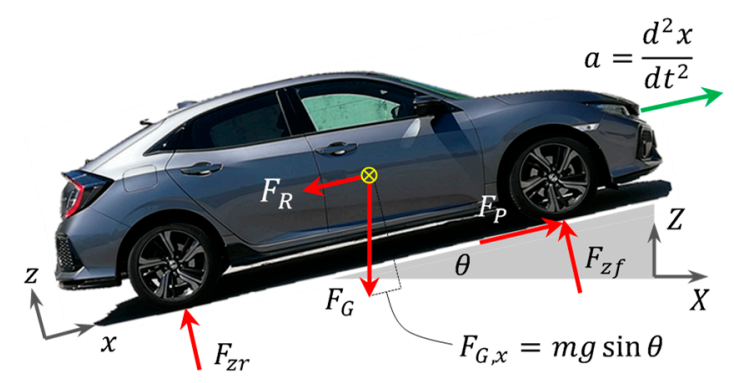

Figure 3. Free-body diagram of a road vehicle. 


$$
W_{P}^{+}=\int_{0}^{L} F_{P}^{+} d x=W_{R}+W_{B}+\Delta E_{M}
$$

thus,

$$
W_{C P}=W_{R}+W_{B}=\int_{0}^{L} F_{R R}+F_{D} d x+\int_{0}^{L} F_{B} d x, \quad F_{B}=-\operatorname{MIN}\left(0, F_{P}\right) .
$$

From the definition of $\eta_{P}$ (Equation (11)), $1-\eta_{P}$ depicts the fraction of the propulsive energy degraded through dissipative braking $\Phi_{B}$ and ensuing from motion non-uniformity $\Phi_{\widetilde{v}}$, from increased drag and rolling losses.

$$
1-\eta_{P}=\frac{W_{R}-W_{\bar{v}}}{W_{C P}}+\frac{W_{B}}{W_{C P}}=\Phi_{\widetilde{v}}+\Phi_{B}
$$

The energy loss ratio $\Phi_{\bar{v}}$ is a function of the standard deviation $\sigma_{v}$ of the speed time-series, according to Equation (17), valid for a symmetric speed distribution around the mean. For all registered speed profiles, the third central moment of the speed proved to be an irrelevant quantity. In the worst case studied, that of a 37 metric ton truck, $\left\{\bar{v}, \sigma_{v}\right\}=\{70.65,17.07\} \mathrm{km} / \mathrm{h}$, Equation (17), differs from the actual value by no more than $0.2 \%$.

$$
\frac{\Phi_{\widetilde{v}}}{\eta_{P}}=3\left(\frac{\sigma_{v}}{\bar{v}}\right)^{2}\left[1-\frac{C_{R R}\left(\frac{2}{3} \bar{v}\right)}{f_{S M R}(\bar{v})}\right]
$$

For a free-flow motion without braking $\left(\Phi_{B}=0\right)$, even when the drag is the prevailing force, i.e., $f_{S M R} \gg C_{R R}$, the motion efficiency is $\eta_{P}>\left[1+3\left(\sigma_{v} / \bar{v}\right)^{2}\right]^{-1}$, being $\eta_{P}>0.97$ for $\sigma_{v}=0.10 \cdot \bar{v}$. The findings of Llopis-Castelló et al. [52] suggest that $\sigma_{v}$ is usually lower for free-flow traffic, thus on a hilly road, the increase in energy consumption results almost entirely from braking associated with vehicle speed control on the steepest downgrades, particularly with heavy vehicles, and not from motion non-uniformity.

\subsection{Gravity Slope}

From the above, it is clear that the minimum downslope necessary to maintain speed by gravity alone, denoted as gravity slope $s_{G}$, is a relevant quantity, as it implies incipient braking when $s<-s_{G}$. For a given speed $v$, the braking work can be expressed as an energy head $H_{B}$ :

$$
H_{B}\left(s_{G}\right)=\frac{W_{B}}{m g}=-\int_{0}^{L}\left(s+s_{G}\right)^{-} d x,\left(s+s_{G}\right)^{-}=\operatorname{MIN}\left(0, s+s_{G}\right)
$$

The gravity slope is defined as a specific motion resistance, since in highway construction gradients are usually small enough to assume $\sin \theta \cong s$ (Figure 3 ), being computed by:

$$
\begin{gathered}
s_{G}=C_{R R}+\frac{F_{D}}{m g}=C_{R R}+\frac{\rho_{a} C_{D W} A}{2 m g} v^{2}, \\
C_{R R}=\left(C_{0}+C_{1} v\right)_{T_{0}} \times\left[1+k \times\left(T-T_{0}\right)\right] .
\end{gathered}
$$

The rolling resistance coefficient $C_{R R}$ was defined as an affine function of the speed and corrected for the measured ambient temperature $T$. The reference values $T_{0}=25^{\circ} \mathrm{C}$ and $k=-0.008{ }^{\circ} \mathrm{C}^{-1}$ are those considered in ISO 28580. Regarding the wind effect upon the drag force $F_{D}$, Howell et al.'s model [38] was used, with the averaged drag coefficient $C_{D W}$ defined as a function of the wind angle $\varphi$ relative to vehicle axis ( $\varphi=0$, for a frontal wind) in such way that,

$$
\frac{F_{D}}{\rho_{a} A / 2}=C_{D W} v^{2}=\frac{1}{\pi} \int_{0}^{\pi} C_{D}(\psi) v_{R}^{2}(\varphi) d \varphi,
$$


where the resultant air flow velocity $v_{R}$ and the yaw angle $\psi$, for a wind speed $v_{W}$, are:

$$
\begin{gathered}
v_{R}=\left(v^{2}+v_{W}^{2}+2 v v_{W} \cos \varphi\right)^{1 / 2}, \\
\psi=\tan ^{-1}\left(v_{W} \sin \varphi /\left(v+v_{W} \cos \varphi\right)\right) .
\end{gathered}
$$

The air density was obtained through the ideal gas law from the measured temperature and the barometric equation, Equation (24) [53], used to compute the local atmospheric pressure $p$ for the road elevation Z.

$$
\frac{p}{p_{0}}=\left(1-\frac{Z}{44330}\right)^{5.257}, p_{0}=101.325 \mathrm{kPa}
$$

\subsection{Horizontal Alignment Efficiency}

As previously stated, the quantity $W_{\bar{v}}$ in Equation (12) is not the minimum conceivable work on a geodesic straight path. In this regard, any straight-line quantity $Y$ will be referred as $Y^{\prime}$. The lack of linearity of a road link is isolated by comparing $W_{\bar{v}}$, for the length $L$, with the minimum work on a straight line, denoted as $W_{\bar{v}^{\prime}}^{\prime}$ for the actual travel time. Knowing the link sinuosity index $S$,

$$
S=L / L^{\prime} \geq 1
$$

it is possible to obtain the link propulsive efficiency $\eta_{L P}$ through the product of the horizontal alignment efficiency $\eta_{H A P}$ by the propulsive efficiency:

$$
\begin{gathered}
\eta_{L P}=\eta_{H A P} \times \eta_{P}, \\
\eta_{H A P}=\frac{W_{\bar{v}}^{\prime}}{W_{\bar{v}}}=\frac{s_{G}(\bar{v} \prime)}{s_{G}(\bar{v})} \times S^{-1} .
\end{gathered}
$$

The $\eta_{H A P}$ quantity reflects simultaneously the decrease of the specific motion resistance, at the reduced straight-line speed $\bar{v}^{\prime}=\bar{v} / S \leq \bar{v}$, and the decrease in traveled distance on the straight-line ideal road, the corresponding road work being,

$$
W_{\text {ideal }}^{\prime}=W_{\bar{v}} \eta_{H A P}+\Delta E_{M}
$$

\subsection{The Free-Flow Mobility Principle}

In free-flow traffic conditions, the vehicle motion is not constrained by headway distance, with its speed governed either by engine power capacity, the case of heavy trucks travelling on hilly roads, or by the driver's free will. For energy rating purposes, standard driving cycles are needed, per vehicle class, in order to compare objectively different road alignments in the design stage. In this regard, the driving proposed favours mobility, i.e., the reference driver must adhere to the road speed limits SL, as far as the vehicle power capacity permits. However, for slopes greater than the vehicle gradeability $s_{V G}$ at the maximum cruising speed $V$ some speed loss is inevitable. This quantity is relevant, being computed for a propulsive power equal to $90 \%$ of the engine rated power $\dot{W}_{r t}$,

$$
s_{V G}(v)=0.9 \frac{\dot{W}_{r t}}{m g v}-s_{G}(v) .
$$

When $s>s_{V G}(V)$, the proposed driving cycle DC is the simplified equilibrium speed SES. The simplification consists (i) on the assumption of a constant specific motion resistance equal to $s_{G}(V)$, and (ii) on the presumption of full power operation on slopes steeper than the vehicle gradeability $s_{V G}(V)$, in accordance with the mobility principle. From this assumption, the SES line $v_{\text {SES }}$ can be 
expressed as a function of the road slope $s$ by Equation (30), being the DC speed line $v_{\text {DC }}$ equal to $v_{\text {SES }}$ bounded by the road speed limits $v_{\mathrm{SL}}$ :

$$
\begin{gathered}
v_{\mathrm{DC}}=\operatorname{MIN}\left(v_{\mathrm{SES}}, v_{\mathrm{SL}}\right), \\
\frac{v_{\mathrm{SES}}}{V}=\left\{\begin{array}{cc}
1, & s \leq s_{V G} \\
\frac{s_{V G}(V)+s_{G}(V)}{s+s_{G}(V)}, & s>s_{V G}
\end{array} .\right.
\end{gathered}
$$

\section{Methodology}

\subsection{Vehicles Studied}

The most frequent vehicle classes found on Portuguese motorways are medium size passenger cars (light-duty vehicles, LDV) and articulated 5-axle heavy-duty trucks (HDTs). Each class was further subdivided in two: the LDV-P and LDV-D, denoting the fleet average EURO 6 petrol and diesel passenger cars, respectively, used in the PHEM emissions model [54]; and the fully loaded and empty trucks, denoted respectively as HDT-F and HDT-E. Table 1 summarizes the principal vehicle specifications, retrieved from Sturm and Hausberger [55] and Rexeis et al. [54], with exception of $C_{0}$ and $C_{1}$ [31]. The truck drag coefficient presented is actually the wind averaged coefficient $C_{D W}$ computed from the directional drag $C_{D}(\psi)$ presented in Rexeis et al. [56] through SAE J1252 (for a $3 \mathrm{~m} / \mathrm{s}$ wind). For the passenger cars, this effect was disregarded, in accordance with the findings of Howell et al. [38]. The test-car specifications reflect manufacturer data.

Table 1. Major specifications of the vehicles studied.

\begin{tabular}{|c|c|c|c|c|c|c|c|c|c|c|}
\hline \multirow{2}{*}{$\begin{array}{l}\text { Vehicle } \\
\text { Classes }\end{array}$} & \multirow{2}{*}{$\begin{array}{c}m \\
{[\mathrm{~kg}]}\end{array}$} & \multirow{2}{*}{$\begin{array}{c}\dot{W}_{r t} \\
{[\mathbf{k W}]}\end{array}$} & \multirow{2}{*}{$\begin{array}{c}A \\
{\left[\mathrm{~m}^{2}\right]}\end{array}$} & \multirow{2}{*}{$C_{D 0}$} & \multicolumn{2}{|c|}{$C_{R R \text { at } 25^{\circ} \mathrm{C}}$} & \multirow{2}{*}{$\begin{array}{c}\eta_{t r} \\
{[\%]}\end{array}$} & \multirow{2}{*}{$\begin{array}{c}V \\
{[\mathrm{~km} / \mathrm{h}]}\end{array}$} & \multirow{2}{*}{$\begin{array}{c}s_{V G}(V) \\
{[\%]}\end{array}$} & \multirow{2}{*}{$\begin{array}{c}s_{G}(V) \\
{[\%]}\end{array}$} \\
\hline & & & & & $C_{0}$ & $C_{1}[\mathrm{~s} / \mathrm{m}]$ & & & & \\
\hline Test-Car & 1307 & 134 & 2.19 & 0.28 & & & \multirow{5}{*}{92} & \multirow{3}{*}{120} & 23.6 & 4.64 \\
\hline LDV-P & 1350 & 84 & 2.14 & 0.30 & $8.00 \times 10^{-3}$ & $2.07 \times 10^{-4}$ & & & 12.5 & 4.68 \\
\hline LDV-D & 1675 & 101 & 2.27 & 0.30 & & & & & 12.4 & 4.22 \\
\hline HDT-F & 37000 & 320 & 9.00 & 0.58 & $5.80 \times 10^{-3}$ & \multirow[b]{2}{*}{0} & & \multirow[b]{2}{*}{90} & 2.06 & 1.11 \\
\hline HDT-E & 15000 & 320 & 9.00 & 0.58 & $7.30 \times 10^{-3}$ & & & & 5.79 & 2.04 \\
\hline
\end{tabular}

Obs.: Gradeability and gravity slopes computed for an air density of $1.18 \mathrm{~kg} / \mathrm{m}^{3}$ (at sea level and $25^{\circ} \mathrm{C}$ ).

Figure 4 shows the eastbound (EB) driving cycles as red lines, between the A25 junctions with the IC2 and A24. For the LDV classes, the speed limit line is used, upper red line, and for the HDT, the SES line is proposed, Equation (30). The heavy black lines are measured speed profiles, to be discussed later. The upper line reflects various LDVs and the other a 37-ton 5-axle truck, a Mercedes-Benz Actros $18444 \times 2$ tractor pulling a $22 \mathrm{~m}^{3}$ water tank (Table 1 ).

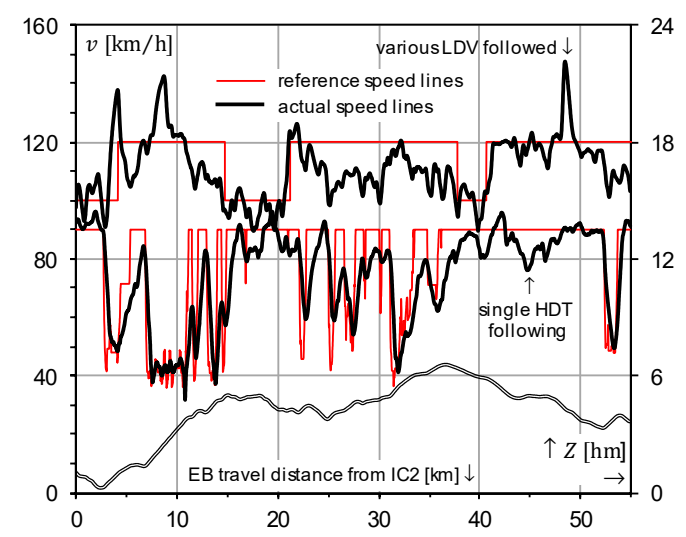

Figure 4. Reference driving-cycles and actual measured speed profiles.

The adherence of the truck speed to the respective reference speed line is more evident, since its movement is highly influenced by power capacity and cruise control governor, contrasting with 
light-duty vehicles speed line, more dependent on the driver's free will. The HDT overall average speed over this road was $70.7 \mathrm{~km} / \mathrm{h}$, with a corresponding average SES of $72.4 \mathrm{~km} / \mathrm{h}$, a small difference. Additionally, studying consecutive road segments $5 \mathrm{~km}$ long, it is concluded that the average SES has a MAE and RMSE of 3.42 and $3.87 \mathrm{~km} / \mathrm{h}$, respectively. These results are fairly good; however, the vehicle was not driven at full power, even on the steepest grades, since the time-averaged engine power over grades steeper than the truck gradeability at $90 \mathrm{~km} / \mathrm{h}$ equals $274 \mathrm{~kW}$, or $86 \%$ of the rated power. The proposed SES line is intended as a road based driving cycle, to be used only in the absence of detailed speed profiles complying with the mobility principle. Such speed lines can only be obtained through microsimulation. However, a purely computational approach is opaque to the user, as it does not explicitly correlate the gradient profile with the computed speed line, opposing Equation (30).

\subsection{Energy-Efficiency Rating}

The propulsive efficiency concept compares the energy consumption on the actual road with that conceivable on an ideal path for the minimum real-world travel time: constrained either by the regulatory speed limits or by vehicle mobility/rated power. A constant speed assumption, CSA, is considered in order to allow a straightforward algebraic approach to the problem. The CSA confines the applicability of the procedure to free-flow conditions. The methodologic approach is described in the flow diagram of Figure 5.

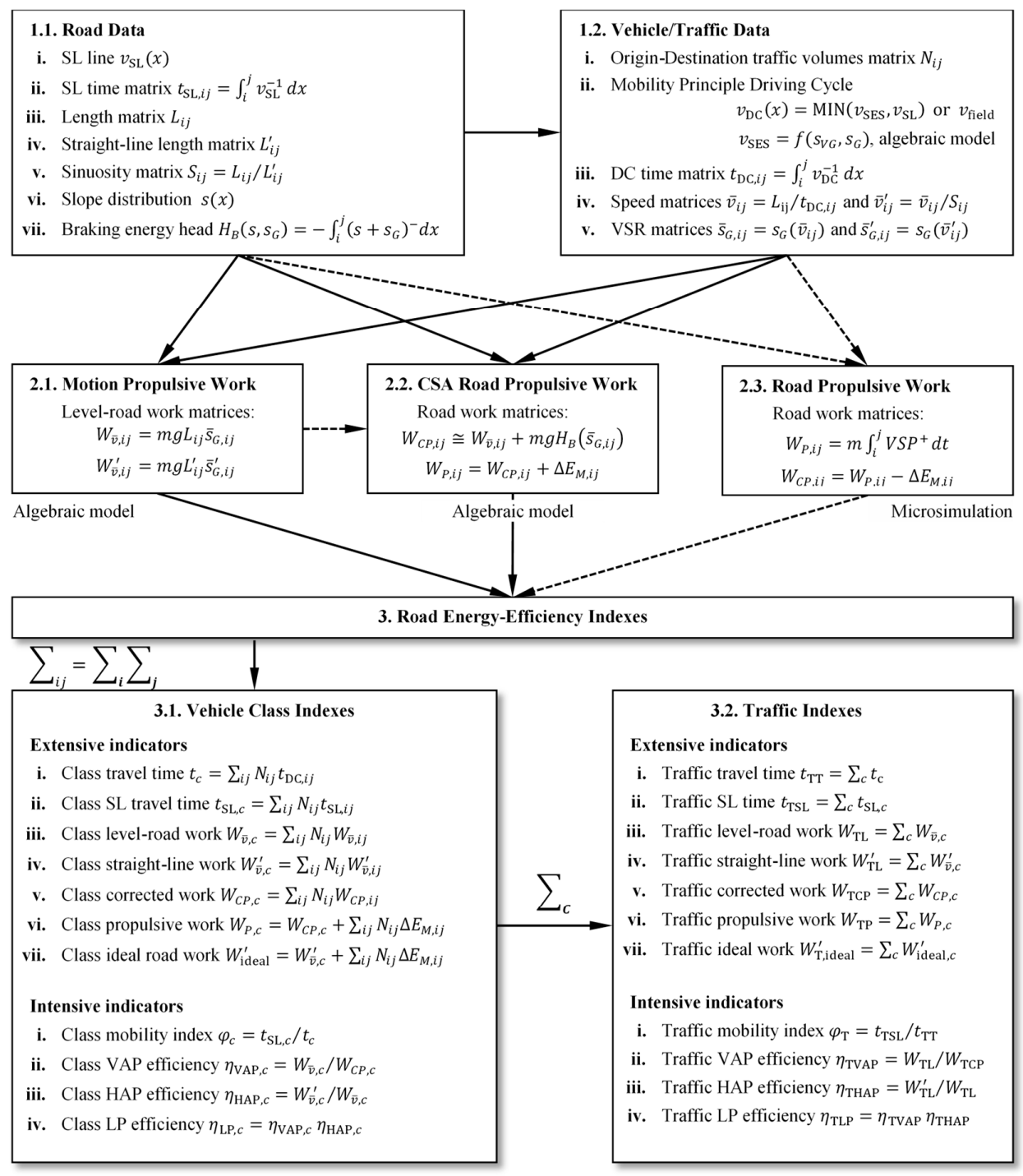

Figure 5. Flow chart of the energy-efficiency rating methodology for free-flow traffic conditions. 
Three levels are shown: the first presents the road and vehicle/traffic data; the second, the data processing needed to obtain the road propulsive energy; and the third, the vehicle/traffic energy-efficiency rating. In step 3.1, the extensive indicators of a given vehicle class $c, Y_{c}$, time or work related, are obtained through the Frobenius product, Equation (31), by means of weighing the road link quantity $Y_{i j}$ by the respective origin-destination traffic volume $N_{i j}$, in vehicles per unit time. The traffic totals $Y_{T}$ in step 3.2 are obtained through the summation of the class extensive quantities, $Y_{T}=\sum_{c} Y_{c}$, where,

$$
Y_{c}=\operatorname{tr}\left(\mathbf{Y} \mathbf{N}^{\mathbf{T}}\right)_{c}=\sum_{i} \sum_{j} N_{i j} Y_{i j}
$$

The intensive indexes in step 3 are the propulsive efficiencies of the vertical and horizontal alignments, $\eta_{V A P}$ and $\eta_{H A P}$, and the link propulsive efficiency $\eta_{L P}$, previously defined. It is recalled that the implicit ideal and real movements have the same duration. In this regard, the traffic flow level is characterized by the mobility index $\varphi_{c}$, defined as the ratio between the minimum conceivable driving time at the road speed limits, $t_{\mathrm{SL}, c}$, and the minimum possible under free-flow conditions according to vehicle class mobility $t_{c}$,

$$
\varphi_{c}=t_{S L, c} / t_{c} \text {. }
$$

All matrices have zero diagonals, with the following exceptions: $S_{i i}=1, \bar{v}_{i i}=v_{\mathrm{SL}}\left(x_{i}\right)$, $\bar{s}_{G, i i}=s_{G}\left(\bar{v}_{i i}\right)$, the same applying to the straight-line corresponding quantities. The above indicators can be expressed according to traffic direction, using the superior triangular matrix of $\mathbf{N}$ for the ascending direction $(j>i)$, and the inferior triangular matrix for the descending direction. The dashed lines identify the microscale calculus sequence ensuing from measured $v_{\text {field }}$, or driving cycles obtained through microsimulation. In such cases, the energy consumption assessment in step 2.3 is necessarily computational. It should be noted that the SES line, Equation(30), allows the entire procedure to be purely algebraic, since the link average speeds $\bar{v}_{i j}$ and the braking energy head $H_{B}$ (a road characteristic) can be algebraically modeled, being dependent on the vehicle gradeability and gravity slope.

\section{Validation}

\subsection{Testing Grounds}

The CSA procedure was validated through a VSP analysis applied to the speed profiles obtained on the controlled access highway A25, under free-flow traffic conditions. The tests were conducted between the junctions with the roads IC2 and A24. This segment, $55 \mathrm{~km}$ long, has a ruling gradient of $6 \%$, an overall average slope of $0.48 \%$, eastbound, and an average elevation of $417 \mathrm{~m}$, ranging from 29 to 660 metres. Figure 6 shows the road alignments. The gradient profile was obtained from detailed design data supplied by ASCENDI, the road concessionaire. The data was studied on a second-by-second basis, as usual in a VPS analysis. The field speed profiles were obtained by a $20 \mathrm{~Hz}$ GPS device, a Racelogic VBOX, and smoothed through a robust exponential method [57].

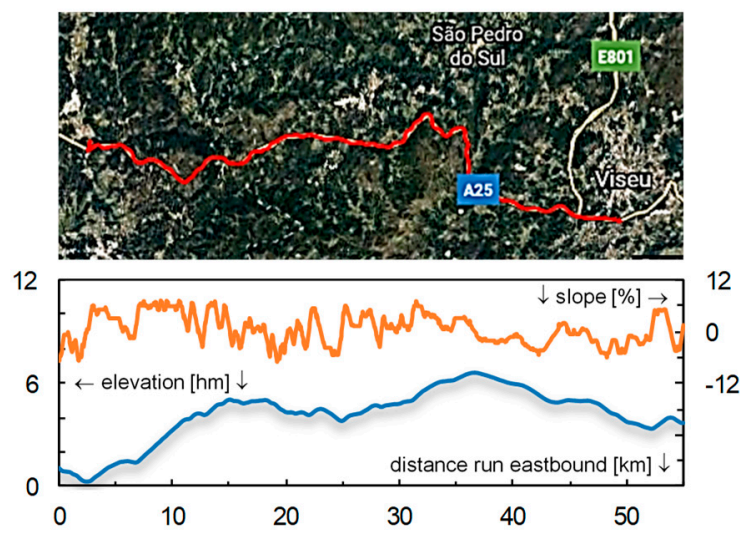

Figure 6. Horizontal and vertical alignments of the Portuguese controlled access highway A25. 


\subsection{Driving Protocols}

To test the CSA influence upon propulsive work for different driving behaviours, uneven fast and slow movements were probed in accordance with two driving protocols: the high speed filtered, HSF, and the low speed filtered, LSF, targeting light-duty vehicles and heavy-duty vehicles, respectively. Figure 7 shows the obtained speed profiles.

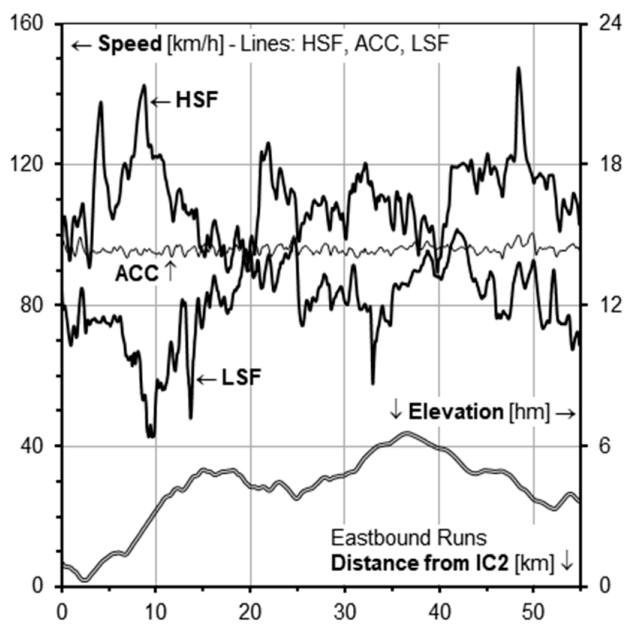

(a)

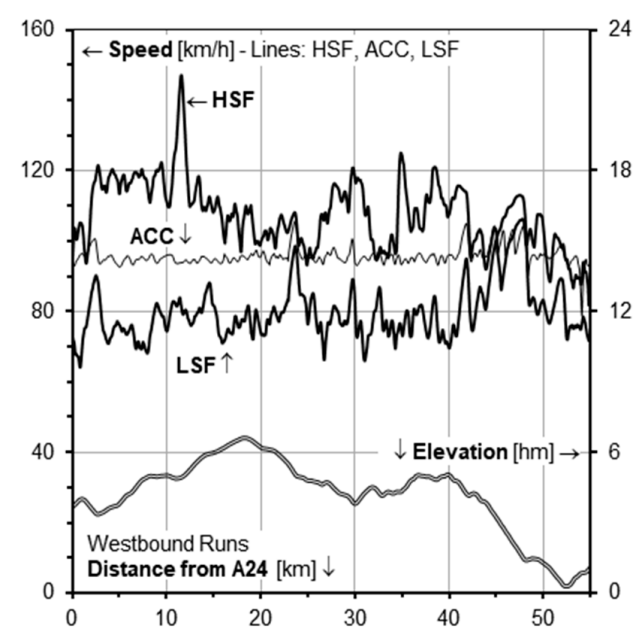

(b)

Figure 7. Speed profiles of the driving protocols LSF (low speed filtered), HSF (high speed filtered); and ACC (adaptive cruise control), for the: (a) eastbound runs; (b) westbound runs.

In the HSF scenario, the driver of the test car followed any vehicle that passed it. For safety reasons, when the speed exceeded the road speed limits by $25 \mathrm{~km} / \mathrm{h}$, the procedure was discontinued, and the next fastest vehicle followed. In the LSF protocol, the driver always kept pace with the slowest vehicles found on the road. In both cases, a minimum headway distance of one hectometer was imposed, to avoid travelling in the wake of the leading vehicle or affecting the driver's behaviour. The strict adherence to the HSF protocol proved to be difficult without disregarding safety. However, since the objective was to obtain uneven speed profiles, the procedure served its purpose. Other runs were made at near constant speed using the adaptive cruise control ACC of the test-car, in order to: (i) infer its viability on a hilly and winding road, and (ii) to assess its adherence to the theoretical CSA performance. The unevenness of measured free-flow speeds is quite marked, thus challenging the proposed CSA model. The probe vehicle was a Honda Civic 1.5 VTEC turbo, 10th generation, with a curb mass of $1307 \mathrm{~kg}$ and a rated power of $134 \mathrm{~kW}$. The gearshift strategy adopted was to downshift when the engine speed fell below $1500 \mathrm{rpm}$ upshifting above $2000 \mathrm{rpm}$.

\subsection{Test-Car Results}

Table 2 shows the main statistics of the driving protocols described. The run designation identifies the travel direction, eastbound (EB) or westbound (WB). The weather conditions are stated also: the temperatures presented are the mean values registered by a Pico TC-08 USB data logger, and the wind force, complying with the Beaufort scale, was obtained from nearby weather stations. The mobility index in the fourth column reveals that the HSF runs were made at an average speed of $97 \%$ of the road speed limits, ranging from 100 to $120 \mathrm{~km} / \mathrm{h}$ (Figure 4). The positive propulsive work $W_{P}^{+}$is presented per unit length, being numerically equal to the average traction force. The average engine power $\dot{W}_{A E}^{+}$, 
under load, depends on the propulsive time fraction PTF, the ratio between the time under traction $t_{F_{P}>0}$ and the total running time $t_{T}$. Thus, considering the transmission efficiency $\eta_{t r}$ :

$$
\begin{gathered}
\dot{W}_{A E}^{+}=\frac{W_{P}^{+}}{\eta_{t r} t_{F_{P}>0}}, \\
t_{F_{P}>0}=P T F \times t_{T} .
\end{gathered}
$$

\begin{tabular}{|c|c|c|c|c|c|c|c|c|}
\hline \multirow{2}{*}{ Run } & \multirow{2}{*}{ Weather } & $\bar{v}$ & $\sigma_{v}$ & \multirow{2}{*}{$\begin{array}{l}W_{P}^{+} / L \\
{[\mathrm{~J} / \mathrm{m}]}\end{array}$} & \multirow{2}{*}{$t_{S L} / t_{T}$} & \multirow{2}{*}{$t_{F_{P}>0} / t_{T}$} & \multirow{2}{*}{$\begin{array}{l}\dot{W}_{A E}^{+} \\
{[\mathbf{k W}]}\end{array}$} & \multirow{2}{*}{$\begin{array}{c}V_{F C} / L \\
{[\mathrm{cl} / \mathrm{km}]}\end{array}$} \\
\hline & & \multicolumn{2}{|c|}{$[\mathrm{km} / \mathrm{h}]$} & & & & & \\
\hline LSF-EB & $20^{\circ} \mathrm{C}$, calm & 78.7 & 12.1 & $430(423)$ & 0.696 & 0.798 & $12.8(12.6)$ & $4.60(4.54)$ \\
\hline ACC-EB & $11^{\circ} \mathrm{C}$, light air & 96.1 & 1.3 & $531(505)$ & 0.850 & 0.832 & $18.5(17.6)$ & $5.42(5.18)$ \\
\hline HSF-EB & $17^{\circ} \mathrm{C}$, gentle breeze & 109.9 & 10.3 & $614(596)$ & 0.973 & 0.860 & $23.7(23.0)$ & $6.33(6.13)$ \\
\hline LSF-WB & $14^{\circ} \mathrm{C}$, light air & 79.5 & 8.4 & $345(331)$ & 0.722 & 0.702 & $11.8(11.3)$ & $3.72(3.57)$ \\
\hline ACC-WB & $19^{\circ} \mathrm{C}$, light air & 95.8 & 2.5 & $417(408)$ & 0.871 & 0.735 & $16.4(16.0)$ & $4.35(4.26)$ \\
\hline HSF-WB & $17^{\circ} \mathrm{C}$, gentle breeze & 106.3 & 9.6 & $490(474)$ & 0.966 & 0.752 & $20.9(20.2)$ & $4.96(4.83)$ \\
\hline
\end{tabular}

Table 2. Vital statistics of the test-car runs on A25.

Obs.: The results inside brackets were computed for a corrected air temperature of $25^{\circ} \mathrm{C}$.

The fuel consumption results FC were integrated from the vehicle fuel map [46] and VSP load. The engine speed was computed from the vehicle transmission gear ratios. The VSP approach allows an unbiased analysis, by filtering non controllable variables such as pavement condition, tire temperature, or weather $[21,49]$. The tests were conducted in daytime with the car windows shut and the air conditioning off. To check the dependability of the calculated FC results, readings (in $\mathrm{cl} / \mathrm{km}$ ) were taken from the car onboard computer (OBC), set to zero at beginning of every test. They were registered in sequence at every $2.5 \mathrm{~km}$, approximately, being taken at the moment of a reading level transition $(\mathrm{of} 0.1 \mathrm{cl} / \mathrm{km})$ to avoid truncation errors. This method depicts cumulative results within the vehicle instruments accuracy, checked against careful pump readings with a certified volume accuracy of $\pm 0.5 \%$. Over the $6000 \mathrm{~km}$ test program, the fuel volume was found to be of $4.1 \%$ above that inferred from $\mathrm{OBC}$ readings.

Figure 8 shows the parity between the VSP-Fuel Map results and the test-car OBC readings along the road. The $R^{2}$ correlation coefficients of individual runs validate the road work computed through VSP analysis, but there is evidence of systematic deviations with HSF runs, since the line slopes are dependent on travel direction. However, these runs were made in succession under a NW gentle breeze (of 8 knots), thus favouring the EB movement and increasing the slope of the corresponding parity line, the opposite happening for the WB run. However, balancing the parity lines is possible assuming a longitudinal wind in the VSP model, Equation (4), favouring the EB movement. For a wind speed of $1.9 \mathrm{~m} / \mathrm{s}$ the lines slopes became equal to 1.03, as in the tests conducted under light air conditions. The round trip results with and without wind differ by $0.5 \%$ only, in line with the conclusions of Howell et al. [38]. However, the sensibility of fuel consumption to wind speed for individual runs was found to be $\partial F C / \partial v_{W}=0.033 F C /(\mathrm{m} / \mathrm{s})$. Given the weather conditions, the explanation advanced is highly plausible.
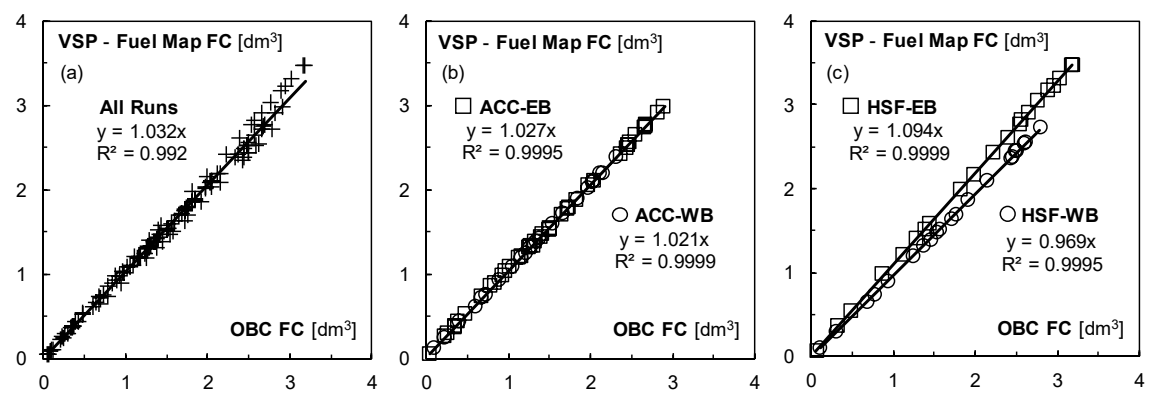

Figure 8. Parity lines between onboard computer $(\mathrm{OBC})$ fuel consumption readings and results computed from the engine fuel map combined and road vehicle specific power (VSP) load. 
Additionally, computed engine operating points were checked against the torque envelope, as shown in Figure 9. The engine has worked at near full throttle only twice, in the sixth gear, while enforcing the HSF protocol in the eastbound run, in the fourth and ninth kilometres (Figure 7). The computed operating points confirm it. The dots below the engine friction line reflect braking events. In free-flow traffic conditions mechanical braking is rare.

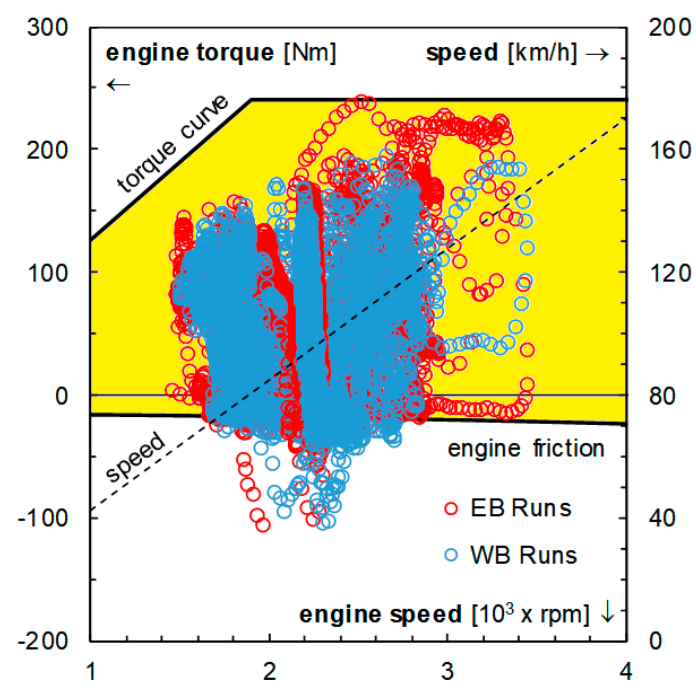

Figure 9. Field engine operating points vs. engine torque envelope. The dashed line depicts the vehicle speed in 6th gear.

\section{Results and Discussion}

\subsection{Results Per Vehicle Class}

Figure 10 shows in a tabular form the main results ensuing from the VSP analysis and the simplified CSA formulation for the LSF, ACC, and HSF speed lines. Results for the major energy-efficiency indexes previously defined are presented for the vehicle classes described in Table 1, namely: the test-car T-C; petrol and diesel cars, LDV-P and LDV-D, respectively; and for the empty and fully loaded truck, HDT-E and HDT-F. The parameters shown are: the propulsive time fraction PTF, Equation (34); the linear propulsive work LPW, per road unit length; the average positive power APP, Equation (33); the fuel consumption FC; the greenhouse gas GHG emissions, obtained from the homologation emission factors of $2.28 \mathrm{~kg}_{\mathrm{CO} 2} / \mathrm{dm}^{3}$ petrol and $2.62 \mathrm{~kg} \mathrm{CO}_{2} / \mathrm{dm}^{3}$ diesel, declared by car builders; the road vertical alignment propulsive efficiency VAPE; the horizontal equivalent HAPE; and the road link propulsive efficiency LPE. Eastbound and westbound movements are analyzed as well as round trips (RT), equivalent to balanced traffic movements. All results consider an air temperature of $25^{\circ} \mathrm{C}$.

The CSA fuel consumptions $V_{F C}$ were obtained through Equation (7) [28], replacing $E_{\mathrm{c}}$ by $\dot{W}_{P}^{+}$ and computing the thermal efficiency at the wheels $\bar{\eta}_{t w}$ through Equation (36), defined from the fuel model (Equation (8)) and considering the transmission efficiency $\eta_{t r}$. The average engine speed $\bar{\omega}$ is obtained in the top gear ratio $r_{t g}$, for the wheel radius $R$ and vehicle speed $\bar{v}$, Equtation (37). The indicated thermal efficiencies considered are $42 \%$ for the direct injection petrol cars, $43 \%$ for the diesel car, and $49 \%$ for the truck $[45,48]$. The expression inside brackets in Equation (36) is limited to the respective optimum brake thermal efficiencies of $37 \%, 40 \%$ and $46 \%$. Equation (35) shows that the fuel consumption can be obtained directly from the propulsive efficiency $\eta_{V A P}$.

$$
V_{F C}=\frac{\dot{W}_{P}^{+}}{\rho_{f} H_{f} \bar{\eta}_{t w}}=\left(\frac{W_{\bar{v}}}{\eta_{V A P}}+\Delta E_{G}\right) \frac{1}{\rho_{f} H_{f} \bar{\eta}_{t w}}
$$




$$
\begin{gathered}
\bar{\eta}_{t w}=\eta_{t r} \times\left(\frac{1}{\eta_{\text {ind }}}+\frac{K_{0} D \bar{\omega}}{\dot{W}_{A E}^{+}}\right)^{-1} \\
\bar{\omega}=\frac{\bar{v} r_{t g}}{R}
\end{gathered}
$$

\begin{tabular}{|c|c|c|c|c|c|c|c|c|c|c|c|c|c|c|c|c|c|c|c|c|c|c|c|c|c|c|c|c|}
\hline \multirow{3}{*}{$\begin{array}{l}0 \\
0 \\
\frac{0}{0} \\
\frac{0}{0} \\
\frac{0}{0} \\
0 \\
0\end{array}$} & \multirow{3}{*}{$\begin{array}{r}\text { Run } \rightarrow \\
\text { Direction } \rightarrow \\
\text { Parameters } \downarrow\end{array}$} & \multicolumn{9}{|c|}{ LSF } & \multicolumn{9}{|c|}{ ACC } & \multicolumn{9}{|c|}{ HSF } \\
\hline & & \multicolumn{3}{|c|}{ Eastbound } & \multicolumn{3}{|c|}{ Westbound } & \multicolumn{3}{|c|}{ Round Trip } & \multicolumn{3}{|c|}{ Eastbound } & \multicolumn{3}{|c|}{ Westbound } & \multicolumn{3}{|c|}{ Round Trip } & \multicolumn{3}{|c|}{ Eastbound } & \multicolumn{3}{|c|}{ Westbound } & Rou & and $T$ & \\
\hline & & के & 改 & 苛 & 离 & 苑 & 苛 & के & 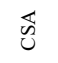 & $\dot{\vec{z}}$ & के & 苑 & 苛 & के & 峁 & $\dot{\vec{z}}$ & $\vec{n}$ & $\overleftrightarrow{⿱ 艹 ⿰ 丿 ㇅ 口 ~}$ & $\dot{\vec{z}}$ & के & 峁 & 苛 & के & 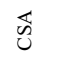 & 苛 & के & 峁 & 节 \\
\hline & PTF [-]: & 0.80 & $0.79-$ & & 0.70 & 0.70 & -0.6 & 0.75 & 0.74 & -0.8 & 0.83 & 0.85 & 1.6 & 0.74 & 0.76 & 3.4 & 0.78 & 0.80 & 2.4 & 0.86 & 0.89 & 3.7 & 0.75 & 0.78 & 4.0 & 0.81 & 0.84 & 3.9 \\
\hline & LPW $[\mathrm{J} / \mathrm{m}]:$ & 423 & 428 & 1.2 & 331 & 351 & 5.8 & 377 & 389 & 3.2 & 505 & 505 & 0.1 & 408 & 414 & 1.6 & 456 & 459 & 0.7 & 596 & $580-$ & -2.8 & 474 & $463-2$ & -2.4 & 535 & & -2.6 \\
\hline & $\mathrm{APP}[\mathrm{kW}]$ & 12.6 & 12.9 & 2.3 & 11.3 & 12.1 & 6.4 & 12.0 & 12.5 & 4.1 & 17.6 & 17.3 & -1.5 & 16.0 & $15.8-$ & -1.7 & 16.9 & $16.6-$ & -1.6 & 23.0 & $21.6-$ & -6.3 & 20.2 & $19.0-$ & -6.2 & 21.7 & & -6.3 \\
\hline$I$ & $\mathrm{FC}[\mathrm{cl} / \mathrm{km}]:$ & 4.54 & $4.53-$ & & 3.57 & 3.78 & 5.7 & 4.06 & 4.15 & 2.4 & 5.18 & 5.23 & 0.9 & 4.26 & 4.37 & 2.8 & 4.72 & 4.80 & 1.8 & 6.13 & $5.90-$ & -3.9 & 4.83 & $4.80-1$ & 0.6 & 5.48 & & -2.4 \\
\hline$\dot{\omega}$ & $\mathrm{GHG}[\mathrm{gCO} / \mathrm{km}]:$ & 104 & $103-$ & -0.2 & 81 & 86 & 5.7 & 92 & 95 & 2.4 & 118 & 119 & 0.9 & 97 & 100 & 2.8 & 108 & 109 & 1.8 & 140 & & -3.9 & 110 & (1) 109 & -0.6 & 125 & 122 & -2.4 \\
\hline & VAPE [\%]: & 90.4 & $89.8-$ & & 84.8 & 80.9 & -3.9 & 87.8 & $85.0-$ & -2.8 & 95.6 & 95.7 & 0.1 & 90.2 & $89.0-$ & -1.2 & 92.9 & $92.2-$ & -0.7 & 95.9 & 98.9 & 3.0 & 90.6 & 93.12 & 2.4 & 93.5 & 96.0 & 2.5 \\
\hline & HAPE [\%]: & 59.4 & 59.4 & & 59.3 & 59.3 & & 59.4 & 59.4 & & 57.2 & 57.2 & - & 57.2 & 57.2 & 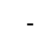 & 57.2 & 57.2 & & 55.8 & 55.8 & - & 56.1 & 56.1 & - & 55.9 & 55.9 & - \\
\hline & LPE [\%]: & 53.7 & $53.3-$ & & 50.3 & 48.0 & -2.3 & 52.1 & 50.5 & -1.6 & 54.7 & 54.7 & 0.1 & 51.6 & 50.9 & -0.7 & 53.1 & 52.7 & & 53.5 & 55.1 & 1.7 & 50.9 & 52.2 & 1.4 & 52.3 & 53.7 & 1.4 \\
\hline & PTF [-]: & 0.80 & 0.79 & & 0.70 & 0.70 & -0.7 & 0.75 & $0.74-$ & -1.1 & 0.83 & 0.85 & 1.6 & 0.74 & 0.76 & 2.8 & 0.79 & 0.80 & 2.2 & 0.86 & 0.89 & 3.9 & 0.75 & 0.78 & 4.1 & 0.81 & 0.84 & 4.0 \\
\hline & LPW $[\mathrm{J} / \mathrm{m}]$ : & 439 & 444 & 1.2 & 344 & 364 & 5.8 & 391 & 404 & 3.2 & 524 & 524 & 0.1 & 423 & 430 & 1.6 & 474 & 477 & 0.8 & 620 & $603-$ & -2.8 & 493 & $481-2$ & -2.4 & 557 & & -2.6 \\
\hline & APP [kW] & 13.0 & 13.4 & 2.8 & 11.7 & 12.5 & 6.5 & 12.4 & 13.0 & 4.4 & 18.2 & 18.0 & -1.5 & 16.6 & $16.4-$ & -1.2 & 17.4 & $17.2-$ & & 23.9 & $22.4-$ & -6.4 & 21.0 & $19.7-$ & -6.32 & 22.5 & & -6.4 \\
\hline 㝏 & $\mathrm{FC}[\mathrm{cl} / \mathrm{km}]:$ & 4.71 & 4.74 & 0.6 & 3.80 & 3.95 & 4.1 & 4.26 & 4.35 & 2.1 & 5.45 & 5.47 & 0.4 & 4.50 & 4.58 & 1.9 & 4.97 & 5.03 & 1.1 & 6.27 & $6.18-$ & -1.5 & 5.09 & $5.03-1$ & -1.1 & 5.68 & 5.60 & -1.3 \\
\hline 0 & $\mathrm{GHG}\left[\mathrm{g}_{\mathrm{CO} 2} / \mathrm{km}\right]:$ & 107 & 108 & 0.6 & 87 & 90 & 4.1 & 97 & 99 & 2.1 & 124 & 125 & 0.4 & 103 & 104 & 1.9 & 113 & 115 & 1.1 & 143 & $141-$ & -1.5 & 116 & $115-$ & -1.1 & 129 & 128 & -1.3 \\
\hline & VAPE [\%]: & 90.6 & $89.9-$ & & 85.1 & 81.2 & -4.0 & 88.0 & $85.3-$ & -2.8 & 95.8 & 95.9 & 0.1 & 90.5 & $89.3-$ & -1.2 & 93.1 & $92.4-$ & -0.7 & 96.0 & 99.0 & 2.9 & 90.9 & 93.3 & 2.4 & 93.7 & 96.2 & 2.5 \\
\hline & HAPE [\%]: & 59.3 & 59.3 & - & 59.2 & 59.2 & - & 59.3 & 59.3 & - & 57.1 & 57.1 & - & 57.1 & 57.1 & - & 57.1 & 57.1 & - & 55.7 & 55.7 & - & 56.0 & 56.0 & - & 55.9 & 55.9 & - \\
\hline & LPE [\%]: & 53.8 & $53.4-$ & & 50.4 & 48.1 & -2.3 & 52.2 & $50.5-$ & -1.6 & 54.7 & 54.7 & 0.1 & 51.7 & $51.0-$ & -0.7 & 53.2 & $52.8-$ & & 53.5 & 55.1 & 1.6 & 50.9 & 52.3 & 1.4 & 52.3 & 53.7 & 1.4 \\
\hline & PTF [-]: & 0.77 & 0.77 & 0.8 & 0.69 & 0.69 & -0.4 & 0.73 & 0.73 & 0.2 & 0.82 & 0.83 & 1.1 & 0.72 & $0.72-$ & -0.3 & 0.77 & 0.77 & 0.5 & 0.83 & 0.87 & 4.5 & 0.73 & 0.77 & 5.4 & 0.78 & 0.82 & 4.9 \\
\hline & LPW [J/m]: & 520 & 527 & 1.3 & 407 & 429 & 5.6 & 463 & 478 & 3.2 & 612 & 612 & -0.1 & 493 & 499 & 1.3 & 552 & 556 & 0.6 & 716 & $694-$ & -3.0 & 567 & $553-2$ & -2.4 & 641 & 624 & -2.8 \\
\hline & $\mathrm{APP}[\mathrm{kW}]$ & 16.1 & 16.2 & 0.5 & 14.1 & 14.9 & 5.9 & 15.1 & 15.6 & 2.9 & 21.7 & 21.4 & -1.2 & 19.8 & 20.2 & 1.6 & 20.8 & 20.8 & 0.1 & 28.7 & $26.6-$ & -7.2 & 24.9 & $23.0-$ & -7.42 & 26.8 & 24.9 & -7.3 \\
\hline 9 & $\mathrm{FC}[\mathrm{cl} / \mathrm{km}]:$ & 4.68 & $4.56-$ & -2.6 & 3.73 & 3.79 & 1.4 & 4.21 & 4.18 & -0.8 & 5.22 & 5.22 & 0.1 & 4.28 & 4.31 & 0.6 & 4.75 & 4.77 & 0.3 & 5.98 & 5.84 & -2.2 & 4.82 & $4.74-1$ & & 5.40 & 5.29 & -1.9 \\
\hline a & $\mathrm{GHG}\left[\mathrm{g}_{\mathrm{CO} 2} / \mathrm{km}\right]:$ & 123 & $120-$ & & 98 & 99 & 1.4 & 110 & 109 & -0.8 & 137 & 137 & 0.1 & 112 & 113 & 0.6 & 124 & 125 & 0.3 & 157 & 153 & -2.2 & 126 & $124-1$ & -1.6 & 141 & 139 & -1.9 \\
\hline & VAPE [\%]: & 88.4 & 87.7 & -0.7 & 81.9 & 78.3 & -3.6 & 85.3 & 82.7 & -2.6 & 93.7 & 94.0 & 0.2 & 87.4 & $86.4-$ & -1.0 & 90.5 & $90.0-$ & -0.5 & 94.3 & 97.5 & 3.2 & 88.3 & 90.62 & 2.4 & 91.5 & 94.1 & 2.6 \\
\hline & HAPE [\%]: & 60.3 & 60.3 & & 60.2 & 60.2 & - & 60.2 & 60.2 & - & 58.0 & 58.0 & - & 58.0 & 58.0 & - & 58.0 & 58.0 & - & 56.6 & 56.6 & - & 56.9 & 56.9 & - & 56.7 & 56.7 & - \\
\hline & LPE [\%]: & 53.3 & $52.9-$ & & 49.3 & 47.1 & -2.2 & 51.4 & $49.8-$ & -1.6 & 54.4 & 54.5 & 0.1 & 50.7 & $50.1-$ & -0.6 & 52.5 & $52.2-$ & & 53.3 & 55.1 & 1.8 & 50.2 & 51.6 & 1.3 & 51.9 & 53.4 & 1.5 \\
\hline & PTF [-]: & 0.70 & $0.69-$ & & 0.64 & 0.65 & 1.3 & 0.67 & $0.67-$ & -0.3 & 0.74 & 0.74 & -0.5 & 0.67 & 0.68 & 1.5 & 0.71 & 0.71 & $\overline{0.5}$ & & & & & & & & & \\
\hline & $\mathrm{LPW}[\mathrm{J} / \mathrm{m}]:$ & 3.94 & 3.98 & 1.2 & 3.02 & 3.16 & 4.8 & 3.48 & 3.57 & 2.7 & 4.49 & 4.47 & -0.3 & 3.56 & 3.59 & 0.9 & 4.02 & 4.03 & 0.2 & & & & & & & & & \\
\hline & $\operatorname{APP}[\mathrm{kW}]$ & 134 & 138 & 3.0 & 113 & 117 & 3.5 & 124 & 128 & 3.1 & 175 & 175 & 0.1 & 154 & $153-$ & -0.5 & 165 & $164-$ & & & & & & & & & & \\
\hline 瓷 & $\mathrm{FC}[\mathrm{cl} / \mathrm{km}]:$ & 30.6 & $30.2-$ & -1.4 & 24.0 & 24.8 & 3.4 & 27.3 & 27.5 & 0.7 & 34.5 & 33.7 & -2.5 & 27.8 & 27.7 & -0.5 & 31.2 & $30.7-$ & & & & & & & & & & \\
\hline 真 & $\mathrm{GHG}[\mathrm{kgCO} 2 / \mathrm{km}]:$ & 0.80 & $0.79-$ & -1.4 & 0.63 & 0.65 & 3.4 & 0.72 & 0.72 & 0.7 & 0.90 & 0.88 & -2.5 & 0.73 & $0.73-$ & -0.5 & 0.82 & $0.80-$ & & & & & & & & & & \\
\hline & VAPE [\%]: & 77.0 & $76.6-$ & -0.4 & 68.2 & 65.7 & -2.5 & 72.6 & $70.7-$ & -1.9 & 84.7 & 85.2 & 0.6 & 75.1 & $74.6-$ & -0.5 & 79.7 & $79.5-$ & & & & & & & & & & \\
\hline & HAPE [\%]: & 60.6 & 60.6 & - & 60.5 & 60.5 & - & 60.5 & 60.5 & - & 57.8 & 57.8 & 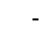 & 57.8 & 57.8 & & 57.8 & 57.8 & & & & & & & & & & \\
\hline & LPE [\%]: & 46.7 & $46.4-$ & & 41.3 & 39.7 & -1.5 & 44.0 & $42.8-$ & -1.2 & 48.9 & 49.2 & 0.3 & 43.4 & $43.1-$ & -0.3 & 46.1 & $46.0-$ & & & & & & & & & & \\
\hline & PTF [-]: & 0.63 & $0.62-$ & -2.3 & 0.57 & 0.56 & -0.6 & 0.60 & $0.59-$ & -1.5 & & & & & & & & & $\mathrm{FF}=\mathrm{P}$ & Propul & Isive $\mathrm{T}$ & te & Fractio & on (unc & der $p$ & oower) & & \\
\hline & LPW $[\mathrm{J} / \mathrm{m}]:$ & 8.08 & 8.13 & 0.6 & 6.01 & 6.19 & 3.0 & 7.04 & 7.16 & 1.6 & & & & & & & & LPV & $\mathrm{W}=\mathrm{L}$ & Linear & Positi & ive $\mathrm{W}$ & Nork (p & per roa & ad len & ngth) & & \\
\hline & $\mathrm{APP}[\mathrm{kW}]$ & 305 & 314 & 2.9 & 254 & 264 & 3.7 & 281 & 290 & 3.2 & & & & & & & & & $P=A$ & Avg. $\mathrm{P}$ & Positiv & ve Po & wer (er & engine) & & & & \\
\hline 站 & $\mathrm{FC}[\mathrm{cl} / \mathrm{km}]:$ & 56.4 & $55.3-$ & & 42.4 & 42.9 & 1.1 & 49.4 & $49.1-$ & -0.7 & & & & & & & & & $\mathrm{C}=\mathrm{F}$ & Fuel C & Consun & mptio & & & & & & \\
\hline & $\mathrm{HG}\left[\mathrm{kgCO}_{2} / \mathrm{km}\right]:$ & 1.48 & $1.45-$ & & 1.11 & 1.12 & 1.1 & 1.29 & $1.29-$ & -0.7 & & & & & & & & & $\mathrm{IG}=\mathrm{C}$ & Greenh & house & Gas & (emiss & sions) & & & & \\
\hline & VAPE [\%]: & 55.2 & 55.4 & 0.2 & 46.1 & 45.1 & -1.0 & 50.5 & $49.7-$ & -0.8 & & & & & & & & VAP & $\mathrm{E}=\mathrm{V}$ & Vertica & al Alig & gnmer & nt Prop & pulsive & e Effic & iciency & & \\
\hline & HAPE [\%]: & 65.7 & 65.7 & & 65.5 & 65.5 & & 65.6 & $65.6-$ & & & & & & & & & HAP & $\mathrm{E}=\mathrm{H}$ & Horizo & ontal A & Alignı & ment $\mathrm{P}$ & Propuls & sive $\mathrm{E}$ & Efficier & ency & \\
\hline & LPE [\%]: & 36.3 & 36.4 & 0.1 & 30.2 & 29.5 & -0.7 & 33.1 & $32.6-$ & -0.5 & & & & & & & & & $\mathrm{E}=\mathrm{L}$ & Link $P$ & Propuls & sive $\mathrm{E}$ & Efficien & ncy & & & & \\
\hline
\end{tabular}

Figure 10. Energy-efficiency rating of the motorway A25 through the VSP and constant speed assumption (CSA) methodologies. The road sinuosity index is 1.28 , and the overall average slope is $0.48 \%$, eastbound.

The propulsive efficiencies of passenger cars on the A25 are high, despite its hilly nature, ranging from $82 \%$, for the LSF WB run with the diesel car, up to $96 \%$ with the petrol car, for the HSF EB run, with round trip results of $85 \%$ to $94 \%$. These results reflect an increase in energy consumption of $\% 7$ to $17 \%$ relatively to flat road travelling, in line with the findings of $[29,49]$. Regarding the heavy-duty truck (HDT), the same cannot be said, with VAPE values varying from $46 \%$, on the WB loaded movement, up to $85 \%$ on the EB run with the empty truck. The lowest efficiencies occur downhill with the heaviest vehicle configurations since the mechanical energy losses through braking are naturally higher in those conditions. The propulsive efficiency of balanced traffic movements (or round trips) with the 
loaded truck configuration is $50.5 \%$. This means that $49.5 \%$ of the propulsive energy is dissipated through braking and motion non-uniformity (increased drag and rolling resistances). The round trip link efficiency of $33.1 \%$ reveals that the corresponding energy consumption on an ideal straight-line would be one-third of the present value, thus disclosing the scale of the road improvement potential. Regarding FC or GHG emissions, a fully loaded HDT is the equivalent to 10 LDV. Therefore, the economic and environmental impact of a road alignment is considerably higher when the traffic of heavy vehicles is significant. As shown, the microscale VSP analysis, based on the actual speed lines, and the simplified CSA model produced similar results. The differences found are small and could be disregarded almost certainly in any study concerning traffic energy consumption, given the stochastic nature of road traffic. The differences are naturally smaller for the ACC runs, since they reflect constant speed operation, in real-world conditions.

The physical meaning of these indicators can be summarized by recalling that the propulsive work of any vehicle will be ultimately degraded to the environment as heat and turbulence (initially). As such, the energy use can be best assessed by determining propulsive losses. Additionally, discriminating those that are unavoidable form those that can be conceptually eliminated is a relevant engineering goal. The latter result from dissipative braking (powertrain friction included), and as such, is conceptually preventable on an ideal transport system. The former ensues from the rolling and aerodynamic drag, and are intrinsic to motion. They can be mitigated by improving the vehicle design (tires included), but are ultimately unavoidable. Its minimum value can be achieved through constant speed driving $[27,28]$. Regarding braking losses, in a congested (urban) traffic scenario, headway distance constrains impose frequent braking. On the other hand, even if free-flow traffic conditions prevail, if a road is hilly braking will be needed to sustain the vehicle speed within safe limits. Therefore, from an energy-efficiency point of view, the rating of a road alignment through a $0-100 \%$ scale should return the value 100 when the propulsive losses are imputable solely to vehicle design and tend to zero when the unavoidable motion energy losses are negligible in relation to the total losses produced on the road. The VAPE index proposed in Section 3.1 satisfies these criteria.

Figure 11 highlights the propulsive losses exhibited on the motorway A25 by the heavy-duty truck (HDT) and the test-car studied (Table 1). For clarity reasons, the analysis considers the gradient profile of the road centerline and constant speed travelling. Additionally, the longitudinal force components are expressed in a dimensionless way, as fractions of each vehicle weight. The shaded orange areas identify the unavoidable motion losses, due to the drag $F_{D}$ and to the rolling resistance $F_{R R}$, and the red areas are the braking losses. It is recalled that when the propulsive force exceeds the intrinsic motion resistance, i.e., $F_{P}>F_{R R}+F_{D}$, the vehicle stores mechanical energy, potential or kinetic. Conversely, when $F_{P} \leq F_{R R}+F_{D}$, its mechanical energy diminishes. The orange areas inside this latter envelope reflect the fraction used to overcome the vehicle inherent motion resistance. However, if braking occurs, potential energy is lost, and with it, a part of the extra uphill road work. The passenger car by exhibiting a higher gravity slope, $s_{G}=\left(F_{R R}+F_{D}\right) / m g$, can travel downhill with minimal brake application (Figure 11). Thus, the uphill and downhill movements balance each other almost entirely. As shown, only a small part of the total propulsive losses exhibited by a conventional HDT are imputable to vehicle design. The vertical alignment efficiency reflects this fraction. 


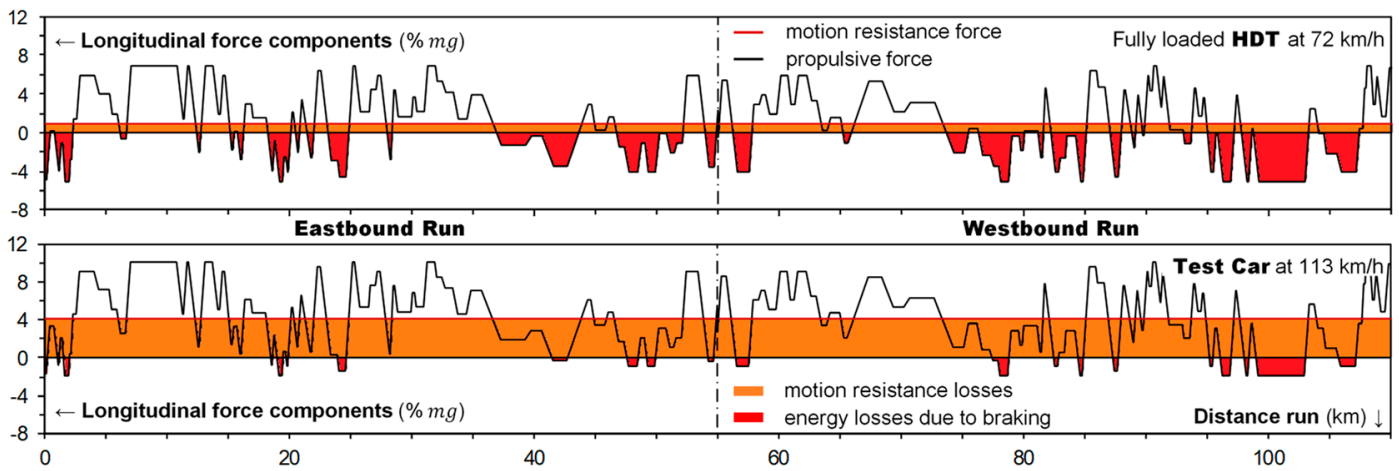

Figure 11. Traction charts of the heavy-duty truck (HDT) and test-car studied highlighting the propulsive energy losses experienced on the motorway A25.

For a given vehicle and origin-destination movement, some propulsive losses intrinsic to motion are inevitable, due to aerodynamic drag and tires performance. These losses depend on road length. The horizontal alignment propulsive efficiency (HAPE) reflects precisely the effect of the link sinuosity upon the minimum conceivable propulsive losses, for the same travel time. Figure 12 portrays graphically this concept. The charts presented are divided in four quadrants (I to IV). The first depicts the normalized straight-line speed $v^{\prime} / \bar{v}$ as a function of the reciprocal of the sinuosity index $S$. The second, shows the motion resistance decrease allowed by the reduce straight-line speed; the third, transports the corresponding normalized straight-line gravity slope $s_{G}^{\prime} / s_{G}$ to the vertical scale of the fourth quadrant, displaying the HAPE index, $\eta_{H A P}$, as a shaded area. As shown, it is proportional to the minimum propulsive work conceivable on an ideal transport system. In Europe, passenger cars are usually allowed to run faster than heavy trucks. Additionally, they exhibit higher values of $A C_{D} / \mathrm{m}$. Thus, its motion resistance is strongly dependent on speed. From the above, it can be concluded that HDTs are particularly affected by the gradient profile and that LDVs and are more sensitive to the road sinuosity, as shown in Figure 10.

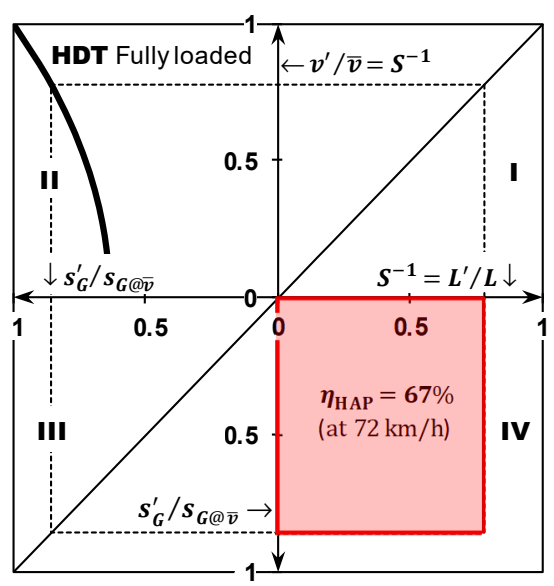

(a)

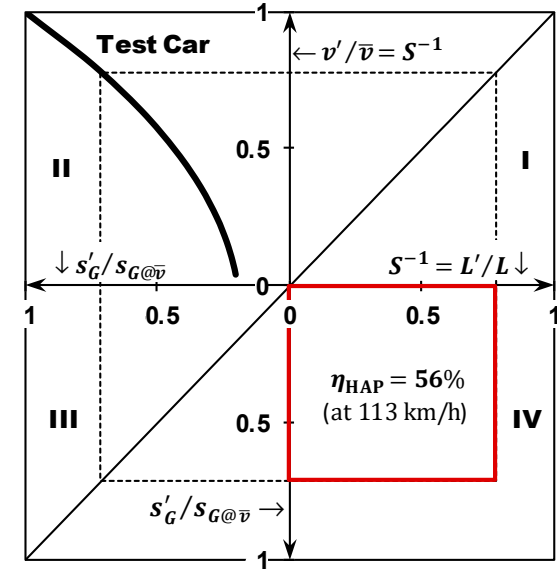

(b)

Figure 12. Horizontal alignment energy-efficiency charts of the vehicles studied highlighting the benefits of straitening the Portuguese motorway A25: (a) heavy-duty truck (HDT); (b) test-car.

Figure 13 presents the fractioning of the test-car propulsive energy through the VSP and CSA methodologies, as a percentage of $m g L$, discriminated by source: PF, being the powertrain friction; $\mathrm{MB}$, mechanical braking; RR, rolling resistance; DR, drag resistance; and KE and GE, kinetic and gravitational energy changes between boundary points. 

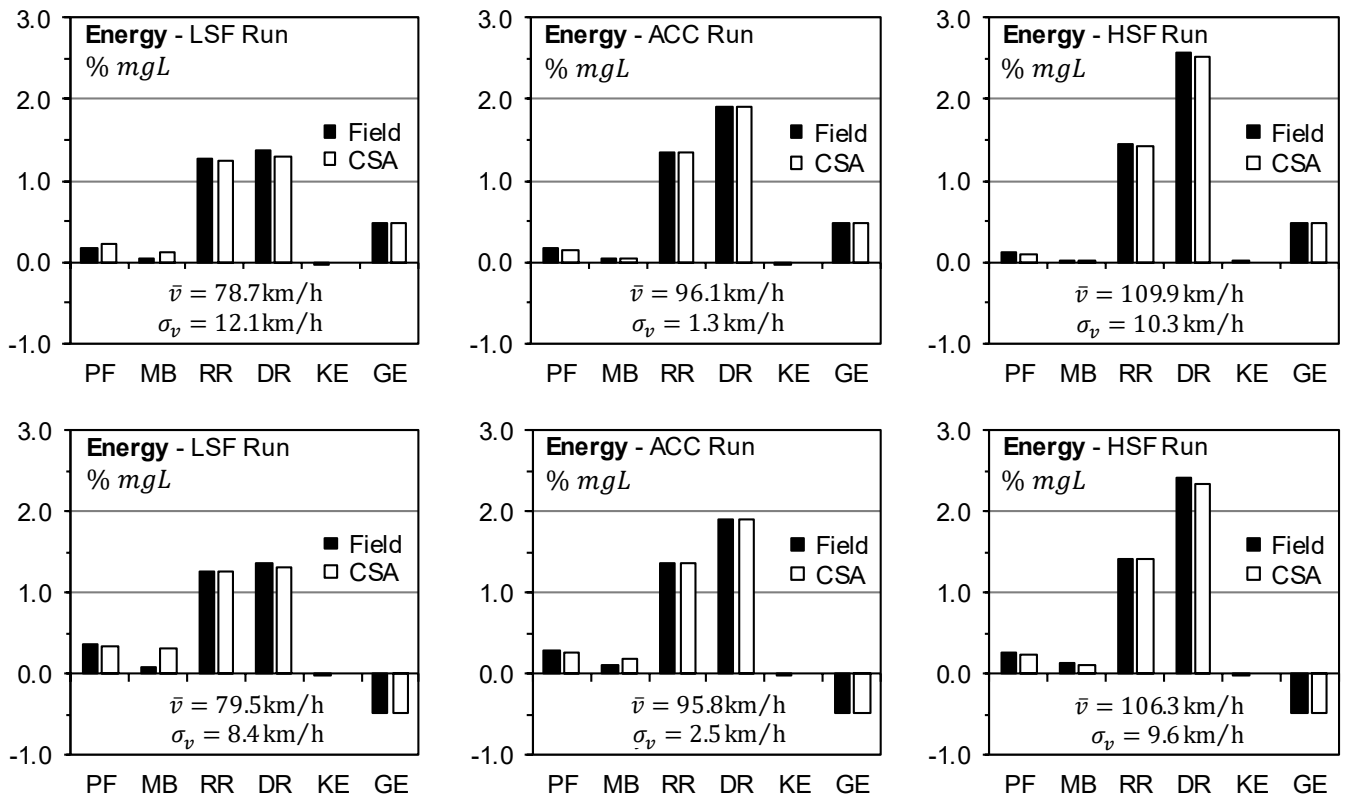

PF, powertrain friction; MB, mechanical braking; RR, rolling resistance;

DR, drag resistance; KE and GE, kinetic and gravitational energy change.

Figure 13. Test car road energy discriminated by source: The upper and lower rows of images depict eastbound and westbound runs, respectively.

The powertrain friction was inferred from the engine fuel map [46]. As shown, the speed non-uniformity increases only slightly the drag or rolling losses, as compared with braking losses. It can also be concluded that in free-flow traffic it is possible to control the vehicle speed mainly by easing or closing the throttle (PF component significantly higher than $\mathrm{MB}$ ). The same level of parity, between CSA and VSP results, was obtained with the HDVs studied, as shown in Figure 14. Figure 14a,b depict eastbound runs. Figure 14a shows the energy balance of a single HDT run (Figure 4) and the others the application of the truck VSP model to the LSF speed profiles. The dissipative braking losses DB are now the prevailing component of the positive propulsive work, being almost exclusively related to downhill braking. The parallelism between the CSA and VSP braking losses proves it since the CSA model does not contemplate acceleration-deceleration cycles.
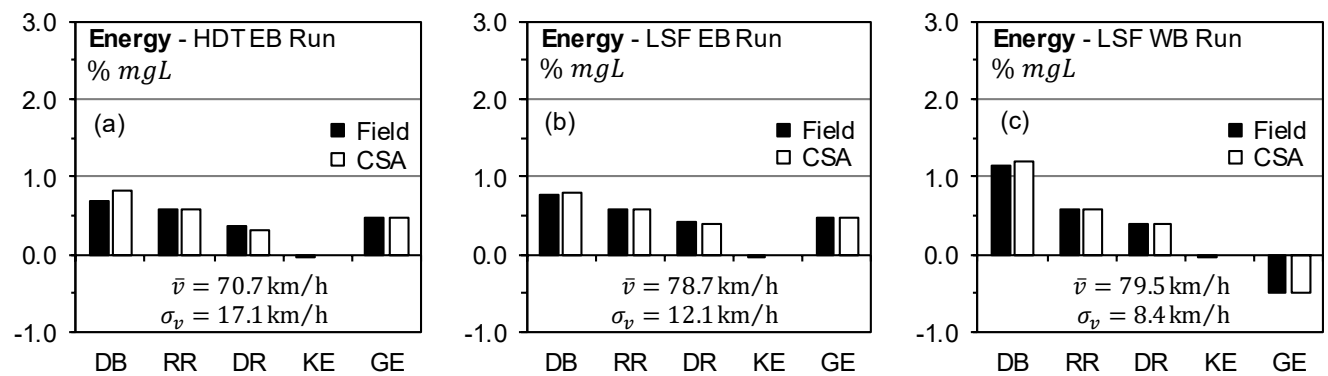

DB, dissipative braking; RR, rolling resistance; DR, drag resistance; KE and GE, kinetic and gravitational energy

Figure 14. Loaded heavy-duty truck (HDT) road energy discriminated by source, reflecting: (a) the HDT run depicted in Figure 4; (b) the eastbound LSF driving protocol shown in Figure 7a; and (c) the westbound LSF protocol (Figure $7 \mathrm{~b}$ ).

\subsection{Effect of Link Length upon CSA Methodology}

The CSA applicability to small road segments or to rolling terrain, as found between kilometres 15 and 30 of A25, Figure 15, was tested subdividing the road into $5 \mathrm{~km}$ segments. The dots depict the 
average sectional results. The black points were computed from the actual speed profiles through VSP analysis, the red dots reflecting CSA results. The parity between booth methodologies along the road is evident, with the exception of a few points concerning the test-car runs, left image, where the divergence is notorious. The reason for this can be traced to the speed bursts resulting from HSF protocol enforcement, Figure 7, that imposed, at moments, near wide-open throttle operation with mixture enrichment, not modeled in Equations (35)-(37). However, these speed bursts reflect particular events and not a natural driving behaviour, thus its significance should not be overstressed.

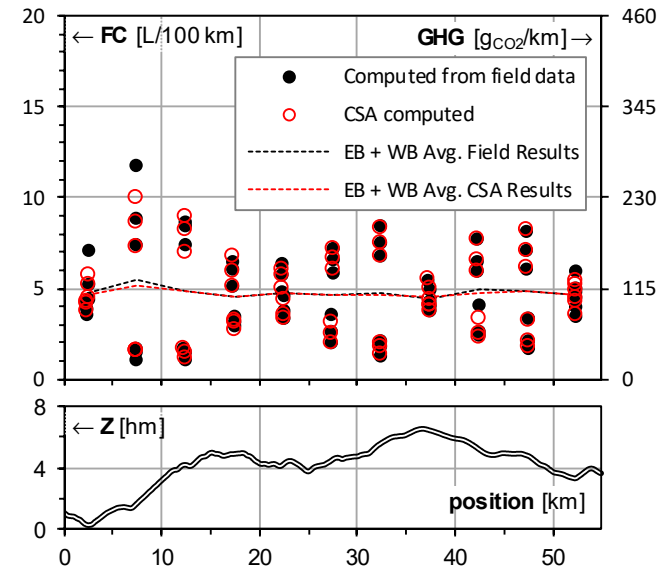

(a)

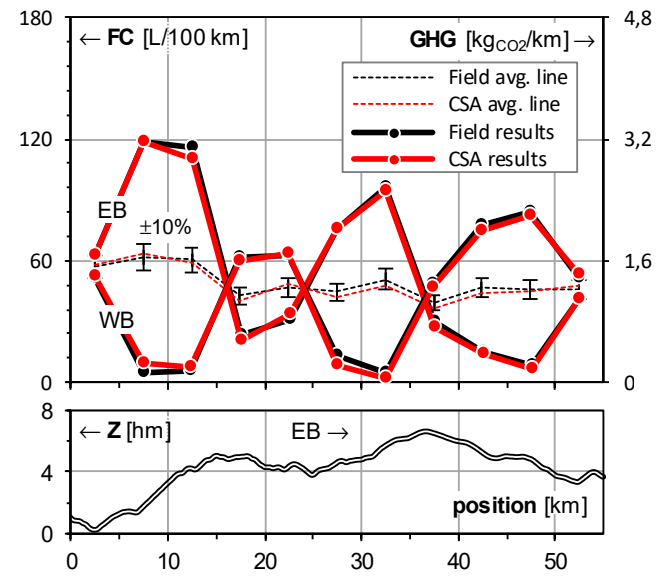

(b)

Figure 15. Fuel consumption and greenhouse gas (GHG) emissions average results for 5-km segments: microscale VSP analysis vs. CSA model. (a) Test-car; (b) Loaded heavy-duty (37-ton) truck.

Traffic results were simulated by averaging the local results of all runs, EB and WB, thus diluting the CSA and VSP divergences, as shown by the dashed lines. The parity between both methodologies was found adequate also for the HDT-F configuration, right image in Figure 15, regardless of the type of terrain encountered. The test car fuel consumption and GHG emissions are almost independent of the vertical alignment of the road, unlike the HDT, dashed lines in Figure 15, due to the near absence of braking events (Figures 9 and 13). In the sectional results presented here, each $5 \mathrm{~km}$ segment was assumed as a road link with boundary speeds equal to those actually observed, in order to compare properly the impact of the constant speed assumption. The parallelism between VSP and CSA results is such that it raised a question similar to the one expressed by Sentoff et al. [32], 'How much road grade matters?', rephrased here as, 'How much the speed line matters?', naturally for free-flow driving conditions on hilly terrain. The answer can be inferred from the cumulative time-series distributions of slopes $s$, normalized accelerations $a / g$, and from the sum of the previous, $s+a / g$, as shown in Figure 16. The similarity between the distributions $a / g$ and $s+a / g$ suggest that (i) the speed and gradient profiles are coupled and (ii) highlight that the magnitude of free-flow accelerations is low, $a /(s g) \ll 1$. The shifting of the acceleration distributions towards negative values when the overall gradient is positive, or the reverse, reinforces point (i). These findings are corroborated by the large-scale field study by Liu et al. [58]. The free-flow power distribution is governed by road grade, thus increasing the CSA methodology representativeness. 


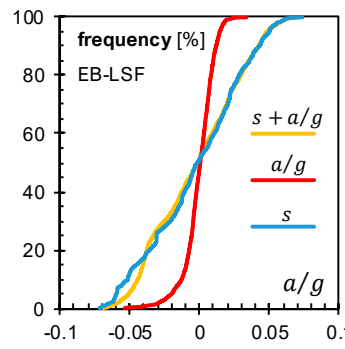

(a)

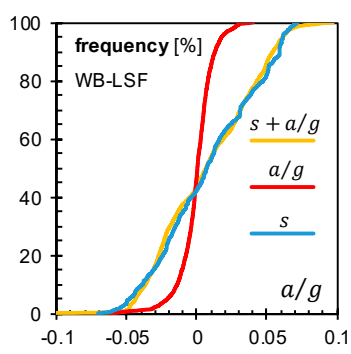

(b)

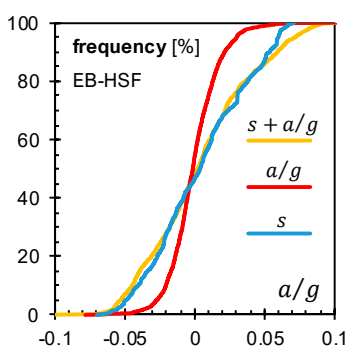

(c)

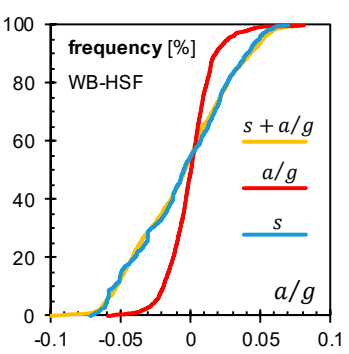

(d)

Figure 16. Cumulative time-series distributions of road slopes $s$, accelerations $a / g$, and its combination $s+a / g$, for the runs: (a) LSF, eastbound; (b) LSF, westbound; (c) HSF, eastbound; (d) HSF, westbound. The low and high speed profiles, LSF and HSF respectively, are shown in Figure 7.

\subsection{Driving Protocol Impact on the HDT Energy Consumption}

The loaded truck results shown in Figure 10 were obtained through VSP analysis for the LSF protocol, when in fact this speed profile reflects the driving behaviour of several HDVs followed, from small trucks to fully loaded heavy trucks, and not a single run. Therefore, in order to assess LSF protocol applicability to the fully loaded HDTs, the following validation procedure was followed: (i) a single run was obtained with a 37-ton truck, black lines in Figure 17; (ii) the LSF speed profile was rescaled to exhibit the same overall average speed of $70.7 \mathrm{~km} / \mathrm{h}$ (as in run i), red lines; and then (iii) the VSP of the same truck configuration was applied to both speed lines and the second-by-second engine power results compared, as well as $5 \mathrm{~km}$ averages regarding FC and propulsive efficiency.

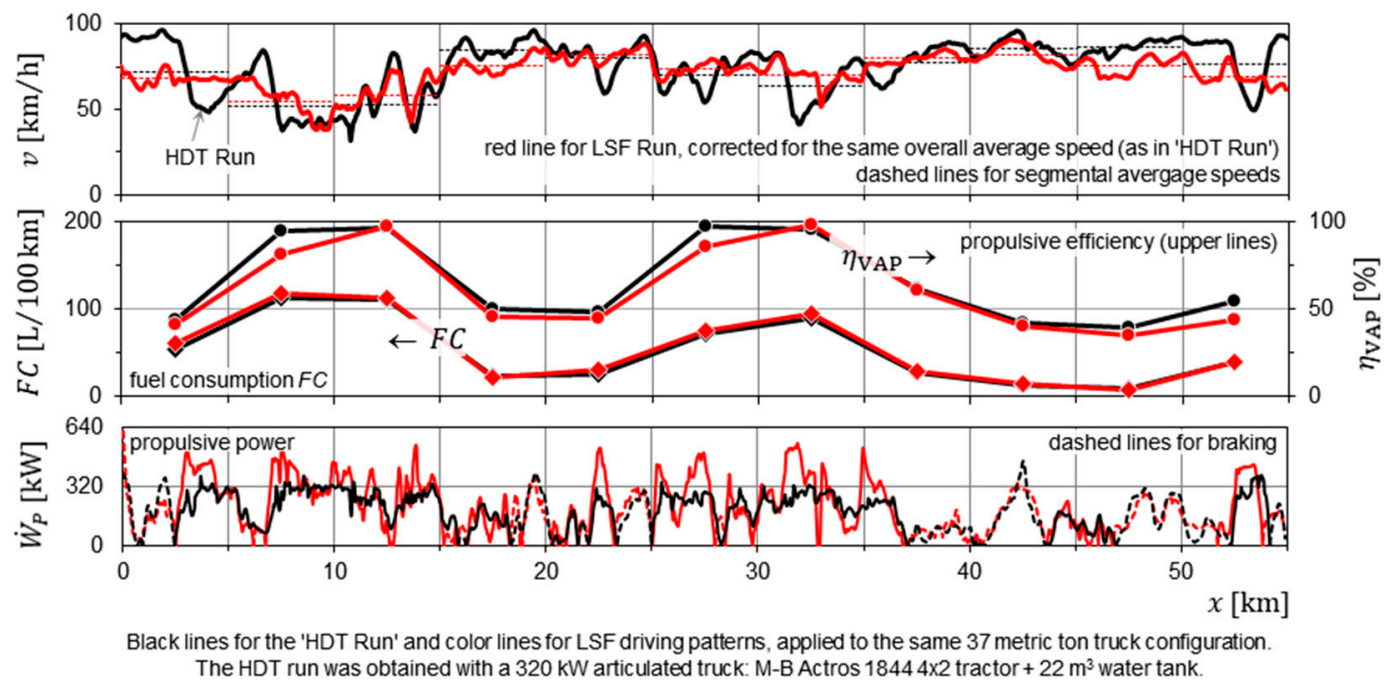

Figure 17. Effect of the driving protocol: LSF driving vs. an actual heavy-duty truck run.

Figure 15 shows that although the instantaneous powers diverge significantly at points, the segmental average results almost overlap each other. The propulsive efficiencies differ somewhat more since they reflect only the motion energy losses and not the gravitational energy change.

\subsection{Margin of Improvement of a Road}

The A25 origin-destination (O-D) traffic volumes are presently unknown. Thus, to illustrate the potential for road improvement as a function of road use (O-D movements), the following scenario was considered: a hypothetical semi-circular road with a $22.5 \mathrm{~km}$ radius and symmetric vertical alignment, having $\pm 4 \%$ slopes, was built on the peninsula shown in Figure 18. The traffic patterns of the vehicle classes described in Table 1 are shown in Figure 19, as well as the road link lengths $\mathbf{L}$ and sinuosity indexes S. The traffic volume considered, of $6750 \mathrm{v} / \mathrm{d}$ per lane, is significantly lower than A25 free-flow 
design value of $8750 \mathrm{v} / \mathrm{d}$ per lane. The HDT traffic is unbalanced. The fractioning of the LDV traffic by fuel type, petrol (P) or diesel (D), is $50-50 \%$. The vehicles are assumed to travel at the respective regulatory speeds $V$, complying in this way with the mobility principle. The exception is the uphill speed of the loaded truck, which was determined from the gradeability equation (Equation (29)), since $s \gg s_{V G}(V)$, being equal to $59 \mathrm{~km} / \mathrm{h}$. The speeds at boundary points are assumed equal. The effect of inertia was neglected since the roads vertical tangents are exceptionally long.

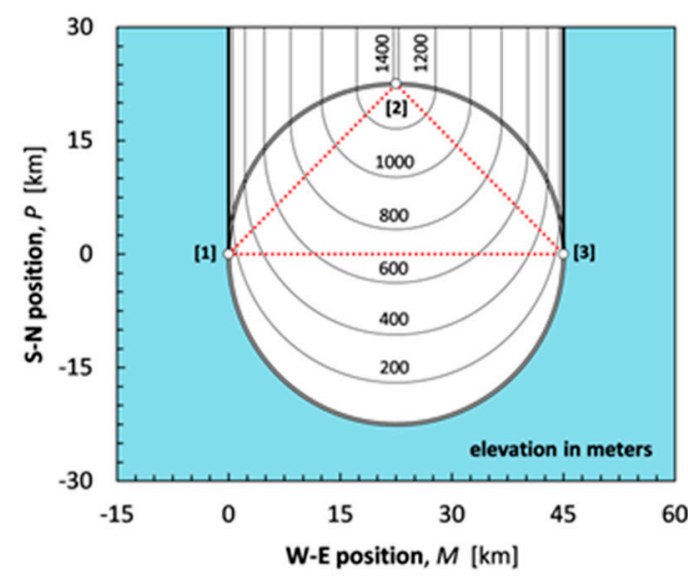

(a)

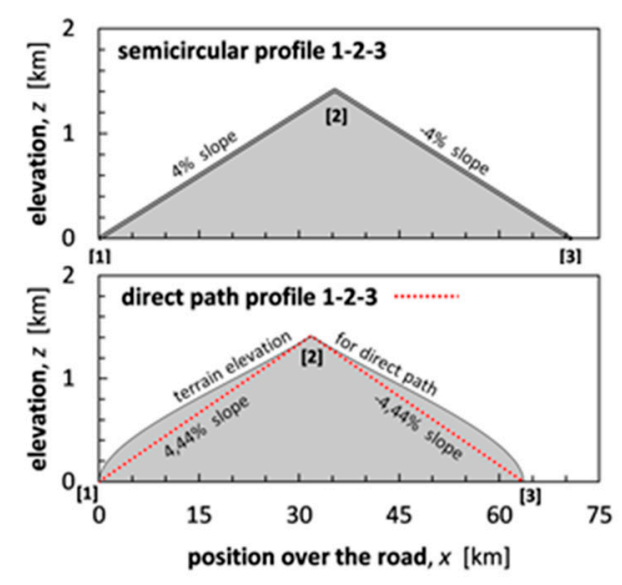

(b)

Figure 18. (a) Topographic chart of a semicircular road and the respective straight-line network, depicted by the dotted red line connecting the nodes [1], [2] and [3]; (b) Elevation profiles.

Traffic volumes and flow patterns:

- Cars

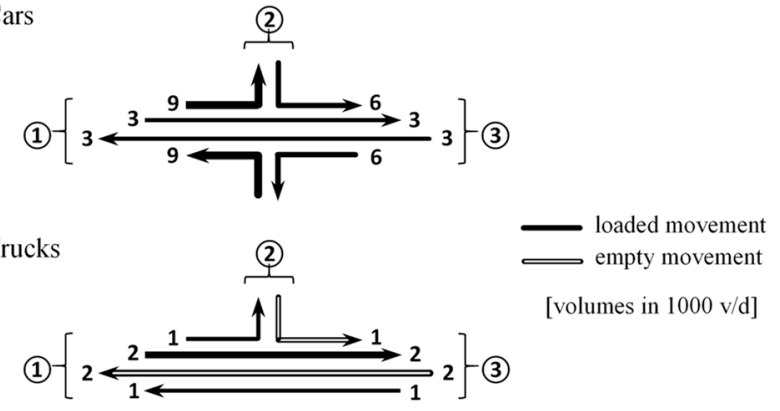

$$
\begin{aligned}
& \mathbf{L}=\left[\begin{array}{ccc}
0 & 1 / 2 & 1 \\
1 / 2 & 0 & 1 / 2 \\
1 & 1 / 2 & 0
\end{array}\right] \cdot 22500 \pi \\
& \mathbf{S}=\left[\begin{array}{ccc}
1 & \frac{\pi \sqrt{2}}{4} & \frac{\pi}{2} \\
\frac{\pi \sqrt{2}}{4} & 1 & \frac{\pi \sqrt{2}}{4} \\
\frac{\pi}{2} & \frac{\pi \sqrt{2}}{4} & 1
\end{array}\right]
\end{aligned}
$$

Figure 19. Traffic volumes for the road depicted in Figure 16, numbers adjacent to the flow lines. The network nodes are encircled. The matrices $\mathbf{L}$ and $\mathbf{S}$ define the link lengths and the sinuosity indexes.

Table 3 presents the main energy-efficiency indexes previously defined. It is recalled that the yearly propulsive work of $405 \mathrm{GWh}$ exceeds its corrected value of $374 \mathrm{GWh}$ due to unbalance traffic movements. The difference of $31 \mathrm{GWh}$ reflects the payload elevation work from the coast to node 2 . The corrected road work represents the propulsive energy required to overcome all dissipative forces acting upon the vehicles. However, only a fraction of it, the propulsive efficiency, can be attributed to the drag and rolling resistance forces, $60 \%$ for this traffic scenario.

The HDTs are more sensitive to the vertical road alignment than LDVs, having a propulsive efficiency of $40 \%$. It is the road, more than the vehicle design, which determines the movement energy efficiency. Regarding LDVs, only incipient braking occurs, thus its propulsive efficiency is close to $100 \%$. In other words, the propulsive energy is due to the vehicle only. Likewise, in a route with downgrades limited by the least observed vehicle gravity slope, no braking actions will occur in free-flow traffic conditions and the propulsive efficiency will be optimal. However, such road design option is not sustainable, even from an energy perspective, if obtained at the expense of an excessive increase in route length, since the vehicle motion resistance is also a dissipative force. The immediate 
conclusion is that comparing routes based merely on the VAPE index is only acceptable for similar road link lengths. Its relevance is to typify the energy consumption per road unit length. On the other hand, the link propulsive efficiency is an absolute index. For the scenario considered, it can be concluded that the road energy consumption could be reduced to $34.6 \%$ of the current value through an ideal transportation system. Such optimal solution would involve using vehicles with regenerative braking capacity and the construction of a straight-line network, as in Figure 18.

Table 3. Efficiency indicators for semicircular route in Figure 18. The results in brackets were computed through the Constant Speed Assumption methodology.

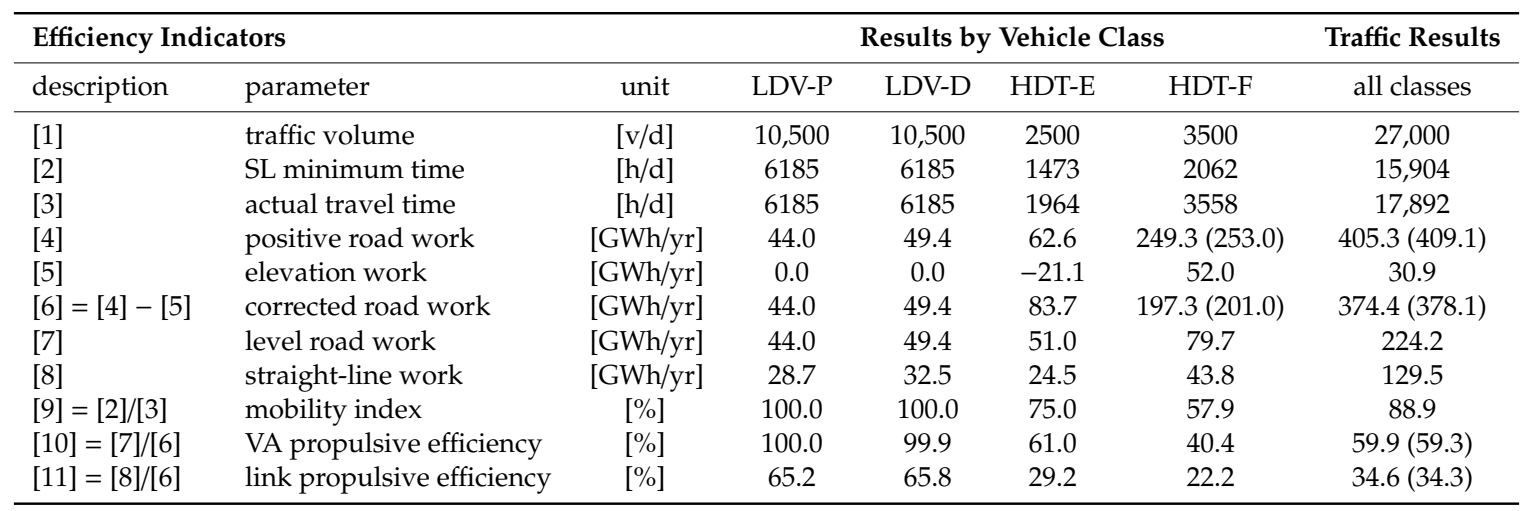

Obs.: The traffic volumes are computed as vehicle-km per day, per km of road length; SL = Speed Limits.

The results in Table 3 can be best interpreted in light of fuel costs and GHG emissions. Table 4 presents such results for a 40-year road alignment life. The full-life energy cost of $94 \mathrm{M€} / \mathrm{km}$ and the environmental impact of $172,000 \mathrm{t}_{\mathrm{CO} 2} / \mathrm{km}$ emphasize the convenience of optimizing such route. In comparison, the cost of the Portuguese motorway network, built between 1999 and 2007, was $3 \mathrm{M} € / \mathrm{km}[59]$.

Table 4. Economic and environmental impact of the route 1-2-3, for a 40-year design life.

\begin{tabular}{|c|c|c|c|c|c|}
\hline \multicolumn{3}{|c|}{ Fuel Costs in Million Euros (Result Per Road Km) } & \multicolumn{3}{|c|}{ GHG Emissions in 1000 kg $_{\mathrm{CO} 2}$ (Result Per Road Km) } \\
\hline Road & Ideal Network & Difference & Road & Ideal Network & Difference \\
\hline $6620(93.7)$ & 2291 & 4330 & $12.1 \times 10^{6}\left(172 \times 10^{3}\right)$ & $4.2 \times 10^{6}$ & $7.9 \times 10^{6}$ \\
\hline
\end{tabular}

The option for a circular route with a direct connection 1-3 along the coastline, Figure 18, would allow rerouting the heavy traffic $N_{13}$ and $N_{31}$ through it. With this alternative, the yearly propulsive work would be reduced from 405 to $284 \mathrm{GWh}$, with a corrected value of $253 \mathrm{GWh}$, thus increasing the network vertical alignment propulsive efficiency from $60 \%$ to $89 \%$. Simultaneously, the traffic mobility increases from $89 \%$ to $93 \%$. These benefits result from the flattening of the vertical alignment available to heavy vehicles.

\subsection{Study Limitations}

The CSA procedure can only by applied to free-flow movements between the entry and exit points of a road link, where the driver is assumed to comply with known local speed limits, thus for classification purposes, this limitation is not relevant. However, the CSA methodology will not produce reliable results if applied to small road segments, between passing points internal to the entire link movement, when the boundary speeds are expected to differ considerably.

Applying the CSA procedure in the design stage of a road requires prior knowledge of the link average speeds, particularly for heavy-duty vehicles. In such cases, the simplified equilibrium speed 
profile, SES line, should be used. However, it is thought that the SES average speed underestimates the true value compliant with the mobility principle, due to vehicle inertia. This aspect can only be assessed through microsimulation. Further research is needed to address this subject.

The proposed rating is intended as an analytical tool, and aims to implement an energy labelling system. It discloses the margin of improvement of the road alignment from an energy perspective, but it is not intended as a public gage of construction quality since it does not consider orographic or urbanistic constrains.

\section{Conclusions}

The proposed methodology characterizes traffic energy-efficiency as a function of the origin-destination movements, or road use, discriminating the vehicle mechanical energy from motion related energy losses. Additionally, it isolates the losses attributable to vehicle design from those resultant from the motion non-uniformity and braking events. The approach followed ensues from basic physical principals, being dimensionally homogenous. Thus, it can be applied to any traffic scenario. However, representative duty-cycles, speed, and gradient based methods, are needed.

Based on the Portuguese A25 case study, a modern road, it was found that the effect of gradient profile upon the motion efficiency of a HDT is very high, as shown by the round-trip vertical alignment propulsive efficiency of 50.5\%, computed from field data and VSP load for a 37-ton truck. Using the CSA algebraic model, this same VAPE index was computed as $49.7 \%$, a very small difference despite the speed unevenness, ranging from 32 to $96 \mathrm{~km} / \mathrm{h}$. The difference to $100 \%$ represents the fraction of the propulsive energy degraded as heat during braking. The cumulative effect of the road sinuosity is reflected on the link propulsive efficiency index. The obtained value of $33.1 \%$, for the actual speed line, or $32.6 \%$ through the CSA methodology, means that the propulsive energy needed along a straight-line path would be reduced to almost one-third of the present value for the same travel time. These results show that the findings of Luin et al. [28] underestimate considerably the relative impact of the road geometry upon HDTs energy consumption, due to the approach limitations discussed in Section 2.1 and resolved in the present study.

The economic and environmental impact of a road alignment, is considerably more dependent on HDV traffic than on LDV traffic, since the energy consumption of passenger cars is only mildly affected by the gradient profile when compared with HDVs, with energy increases in relation to flat road travelling ranging from $7 \%$ to $17 \%$, in line with the findings of other authors [29,49]. Conversely, the LDV traffic energy consumption, and related emissions, are more affected by the road sinuosity than HDV traffic. Regarding the latter, there is evidence that there is more to gain by reducing downhill slopes than uphill gradients. However, this would demand to discriminate the road design per travel direction. This practice is uncommon in Portugal.

This energy-efficiency classification is new and physically meaningful, as it highlights the road margin of improvement from an energy perspective. As shown, it can be obtained adequately through the simplified CSA methodology for free-flow traffic conditions, typical on long distance duty cycles on rural roads. Likewise, it allows the computation of fuel consumption and GHG emissions. In fact, generalized fuel and GHG emissions charts could be constructed as a function of the vehicles gravity slopes and engines thermal efficiencies. The implementation of such charts is a relevant research topic, leading to another: to typify the spatial slope distributions inherent to different types of terrain and construction techniques, in order to develop a useful road design tool, aiming the design of more ecofriendly road networks.

This work confirms the findings of Liu et al. [58], suggesting that free-flow driving cycles cannot be dissociated from the road gradient profile over hilly roads. A relevant conclusion of the present study is the suggestion that road traffic emissions and energy consumption should be based on duty-cycles normalized in a power basis rather than solely on a speed basis, for different vehicle classes. This practice is common in the railway sector. Further research is needed on this subject. 
Author Contributions: H.F.: conceptualization, methodology and writing original draft study; C.M.R.: methodology, validation of the study performed, review and editing of the manuscript; C.P.: methodology, validation of the study performed, supervision, and review and editing of the manuscript. All authors have read and agreed to the published version of the manuscript.

Funding: This research did not receive any specific grant from funding agencies in the public, commercial, or not-for-profit sectors.

Acknowledgments: The authors are indebted to the concessionaire of the Portuguese motorway A25, ASCENDI, for providing the necessary design data for the development of the energy-efficiency rating procedure proposed in this paper, and for the computation of the road's efficiency indicators.

Conflicts of Interest: The authors declare no conflict of interest.

\section{Abbreviations}

$\begin{array}{ll}\text { ACC } & \text { Adaptive Cruise Control } \\ \text { CEFT } & \text { Transport phenomena research center } \\ & \text { (Centro de Estudos de Fenómenos de Transporte) } \\ \text { CITTA } & \text { Research center of territory, transports and environment } \\ & \text { (Centro de Investigação do Território, Transportes e Ambiente) } \\ \text { CMEM } & \text { Comprehensive Modal Emissions Model } \\ \text { CSA } & \text { Constant Speed Assumption, Section 4.2. } \\ \text { DC } & \text { Driving Cycles, Section 3.4. } \\ \text { ELI } & \text { Energy Loss Index, Equations (1)-(3) } \\ \text { FC } & \text { Fuel Consumption (cl/km) } \\ \text { GHG } & \text { Greenhouse Gas (gCO2/km) } \\ \text { HAPE } & \text { Horizontal Alignment Propulsive Efficiency, Section 3.3., Equation (27) } \\ \text { HDT, HDT-E, HDT-F } & \text { Heavy-Duty Truck, Empty HDT, Fully loaded HDT } \\ \text { HDV } & \text { Heavy-Duty Vehicle } \\ \text { HSF } & \text { High Speed Filtered (driving protocol), Section 5.2. } \\ \text { LDV, LVD-D, LVD-P } & \text { Light-Duty Vehicle, Diesel LDV, Petrol LDV } \\ \text { LPE } & \text { Link Propulsive Efficiency, Section 3.3., Equation (26) } \\ \text { LSF } & \text { Low Speed Filtered (driving protocol), Section 5.2. } \\ \text { MAE } & \text { Mean Absolute Error } \\ \text { OBC } & \text { Onboard Computer } \\ \text { O-D } & \text { Origin-Destination (traffic volumes matrix) } \\ \text { PHEM } & \text { Passenger Car and Heavy Duty Emission Model } \\ \text { RMSE } & \text { Root Mean Square Error } \\ \text { SES } & \text { Simplified Equilibrium Speed, Section 3.4., Equation (30) } \\ \text { SL } & \text { Speed Limits (Regulatory) } \\ \text { VAPE } & \text { Vertical Alignment Propulsive Efficiency, Section 3.1., Equations (11)-(13) } \\ \text { VISSIM } & \text { Traffic in cities simulation model } \\ \text { VSP } & \text { (Verkehr In Städten-SIMulationsmodell) } \\ \text { SUMO } & \text { Vehicle Specific Power (W/kg), Section 2.1., Equations (4) and (5) } \\ & \text { Simulation of Urban Mobility } \\ & \\ \text { HA } & \end{array}$

\section{References}

1. Faris, W.F.; Rakha, H.A.; Kafafy, R.I.; Idres, M.; Elmoselhy, S. Vehicle fuel consumption and emission modelling: An in-depth literature review. Int. J. Veh. Syst. Model. Test. 2011, 6, 318. [CrossRef]

2. Bishop, J.D.K.; Stettler, M.E.J.; Molden, N.; Boies, A.M. Engine maps of fuel use and emissions from transient driving cycles. Appl. Energy 2016, 183, 202-217. [CrossRef]

3. Zhou, M.; Jin, H.; Wang, W. A review of vehicle fuel consumption models to evaluate eco-driving and eco-routing. Transp. Res. Part D Transp. Environ. 2016, 49, 203-218. [CrossRef]

4. López-Martínez, J.M.; Jiménez, F.; Páez-Ayuso, F.J.; Flores-Holgado, M.N.; Arenas, A.N.; Arenas-Ramirez, B.; Aparicio-Izquierdo, F. Modelling the fuel consumption and pollutant emissions of the urban bus fleet of the city of Madrid. Transp. Res. Part D Transp. Environ. 2017, 52, 112-127. [CrossRef] 
5. Wang, J.; Rakha, H.A. Fuel consumption model for heavy duty diesel trucks: Model development and testing. Transp. Res. Part D Transp. Environ. 2017, 55, 127-141. [CrossRef]

6. Guang, H.; Jin, H. Fuel consumption model optimization based on transient correction. Energy 2019, 169, 508-514. [CrossRef]

7. Tsiakmakis, S.; Fontaras, G.; Dornoff, J.; Valverde, V.; Komnos, D.; Ciuffo, B.; Mock, P.; Samaras, Z. From lab-to-road \& vice-versa: Using a simulation-based approach for predicting real-world CO2 emissions. Energy 2019, 169, 1153-1165.

8. Hung, W.T.; Tong, H.Y.; Lee, C.P.; Ha, K.; Pao, L.Y. Development of a practical driving cycle construction methodology: A case study in Hong Kong. Transp. Res. Part D Transp. Environ. 2007, 12, 115-128. [CrossRef]

9. Zallinger, M.; Hausberger, S. Measurement of CO2 and Fuel Consumption from Cars in the NEDC and in Real-World-Driving Cycles; Institute for Internal Combustion Engines and Thermodynamics, Graz University of Technology: Graz, Austria, 2009; p. 41.

10. Amirjamshidi, G.; Roorda, M.J. Development of simulated driving cycles for light, medium, and heavy duty trucks: Case of the Toronto Waterfront Area. Transp. Res. Part D Transp. Environ. 2015, 34, 255-266. [CrossRef]

11. Seers, P.; Nachin, G.; Glaus, M. Development of two driving cycles for utility vehicles. Transp. Res. Part D Transp. Environ. 2015, 41, 377-385. [CrossRef]

12. Achour, H.; Olabi, A.G. Driving cycle developments and their impacts on energy consumption of transportation. J. Clean. Prod. 2016, 112, 1778-1788. [CrossRef]

13. Duarte, G.O.; Gonçalves, G.A.; Farias, T.L. Analysis of fuel consumption and pollutant emissions of regulated and alternative driving cycles based on real-world measurements. Transp. Res. Part D Transp. Environ. 2016, 44, 43-54. [CrossRef]

14. Kumar Pathak, S.; Sood, V.; Singh, Y.; Channiwala, S.A. Real world vehicle emissions: Their correlation with driving parameters. Transp. Res. Part D Transp. Environ. 2016, 44, 157-176. [CrossRef]

15. Gallus, J.; Kirchner, U.; Vogt, R.; Benter, T. Impact of driving style and road grade on gaseous exhaust emissions of passenger vehicles measured by a Portable Emission Measurement System (PEMS). Transp. Res. Part D Transp. Environ. 2017, 52, 215-226. [CrossRef]

16. Burton, J.; Walkowicz, K.; Sindler, P.; Duran, A. In-Use and Vehicle Dynamometer Evaluation and Comparison of Class 7 Hybrid Electric and Conventional Diesel Delivery Trucks. SAE Int. J. Commer. Veh. 2013, 6, 545-554. [CrossRef]

17. Liu, K.; Yamamoto, T.; Morikawa, T. Impact of road gradient on energy consumption of electric vehicles. Transp. Res. Part D Transp. Environ. 2017, 54, 74-81. [CrossRef]

18. Yuan, X.; Zhang, C.; Hong, G.; Huang, X.; Li, L. Method for evaluating the real-world driving energy consumptions of electric vehicles. Energy 2017, 141, 1955-1968. [CrossRef]

19. Luin, B.; Petelin, S.; Al-Mansour, F. Microsimulation of electric vehicle energy consumption. Energy 2019, 174, 24-32. [CrossRef]

20. Kamal, M.A.S.; Mukai, M.; Murata, J.; Kawabe, T. Ecological Vehicle Control on Roads With Up-Down Slopes. IEEE Trans. Intell. Transp. Syst. 2011, 12, 783-794. [CrossRef]

21. Park, S.; Rakha, H.; Ahn, K.; Moran, K. Fuel Economy Impacts of Manual, Conventional Cruise Control, and Predictive Eco-Cruise Control Driving. Int. J. Transp. Sci. Technol. 2013, 2, 227-242. [CrossRef]

22. Weißmann, A.; Görges, D.; Lin, X. Energy-optimal adaptive cruise control combining model predictive control and dynamic programming. Control Eng. Pract. 2018, 72, 125-137. [CrossRef]

23. Boriboonsomsin, K.; Barth, M.J.; Zhu, W.; Vu, A. Eco-Routing Navigation System Based on Multisource Historical and Real-Time Traffic Information. IEEE Trans. Intell. Transp. Syst. 2012, 13, 1694-1704. [CrossRef]

24. Ahn, K.; Rakha, H.A. Network-wide impacts of eco-routing strategies: A large-scale case study. Transp. Res. Part D Transp. Environ. 2013, 25, 119-130. [CrossRef]

25. Scora, G.; Boriboonsomsin, K.; Barth, M. Value of eco-friendly route choice for heavy-duty trucks. Res. Transp. Econ. 2015, 52, 3-14. [CrossRef]

26. Zeng, W.; Miwa, T.; Morikawa, T. Prediction of vehicle CO 2 emission and its application to eco-routing navigation. Transp. Res. Part C Emerg. Technol. 2016, 68, 194-214. [CrossRef]

27. Chang, D.J.; Morlok, E.K. Vehicle Speed Profiles to Minimize Work and Fuel Consumption. J. Transp. Eng. 2005, 131, 173-182. [CrossRef] 
28. Luin, B.; Petelin, S.; Al Mansour, F. Modeling the impact of road network configuration on vehicle energy consumption. Energy 2017, 137, 260-271. [CrossRef]

29. Boriboonsomsin, K.; Barth, M. Impacts of Road Grade on Fuel Consumption and Carbon Dioxide Emissions Evidenced by Use of Advanced Navigation Systems. Transp. Res. Rec. 2009, 2139, 21-30. [CrossRef]

30. Zhai, H.; Frey, H.C.; Rouphail, N.M. A Vehicle-Specific Power Approach to Speed- and Facility-Specific Emissions Estimates for Diesel Transit Buses. Environ. Sci. Technol. 2008, 42, 7985-7991. [CrossRef]

31. Rakha, H.A.; Ahn, K.; Moran, K.; Saerens, B.; Van den Bulck, E. Virginia Tech Comprehensive Power-Based Fuel Consumption Model: Model development and testing. Transp. Res. Part D Transp. Environ. 2011, 16, 492-503. [CrossRef]

32. Sentoff, K.M.; Aultman-Hall, L.; Holmén, B.A. Implications of driving style and road grade for accurate vehicle activity data and emissions estimates. Transp. Res. Part D Transp. Environ. 2015, 35, 175-188. [CrossRef]

33. Krajzewicz, D.; Behrisch, M.; Wagner, P.; Luz, R.; Krumnow, M. Second Generation of Pollutant Emission Models for SUMO. In Modeling Mobility with Open Data; Behrisch, M., Weber, M., Eds.; Springer International Publishing: Cham, Switzerland, 2015; pp. 203-221. ISBN 978-3-319-15023-9.

34. Quaassdorff, C.; Borge, R.; Pérez, J.; Lumbreras, J.; de la Paz, D.; de Andrés, J.M. Microscale traffic simulation and emission estimation in a heavily trafficked roundabout in Madrid (Spain). Sci. Total Environ. 2016, 566, 416-427. [CrossRef]

35. Lejri, D.; Can, A.; Schiper, N.; Leclercq, L. Accounting for traffic speed dynamics when calculating COPERT and PHEM pollutant emissions at the urban scale. Transp. Res. Part D Transp. Environ. 2018, 63, 588-603. [CrossRef]

36. Kan, Z.; Tang, L.; Kwan, M.-P.; Ren, C.; Liu, D.; Pei, T.; Liu, Y.; Deng, M.; Li, Q. Fine-grained analysis on fuel-consumption and emission from vehicles trace. J. Clean. Prod. 2018, 203, 340-352. [CrossRef]

37. Jiménez-Palacios, J.L. Understanding and Quantifying Motor Vehicle Emissions with Vehicle Specific Power and TILDAS Remote Sensing. Ph.D. Thesis, Massachusetts Institute of Technology, Cambridge, MA, USA, 1999.

38. Howell, J.; Forbes, D.; Passmore, M. A drag coefficient for application to the WLTP driving cycle. Proc. Inst. Mech. Eng. Part D J. Automob. Eng. 2017, 231, 1274-1286. [CrossRef]

39. Rodrigue, J.-P.; Comtois, C.; Slack, B. The Geography of Transport Systems, 3rd ed.; Routledge: London, UK, 2013; ISBN 978-0-415-82253-4.

40. Franzese, O.; Davidson, D. Effect of Weight and Roadway Grade on the Fuel Economy of Class-8 Freight Trucks (ORNL/TM-2011/471); Oak Ridge National Laboratory: Oak Ridge, TN, USA, 2011.

41. Heywood, J.B. Internal Combustion Engine Fundamentals, 2nd ed.; McGraw-Hill Education: New York, NY, USA, 2018; ISBN 978-1-260-11610-6.

42. Wyatt, D.W.; Li, H.; Tate, J.E. The impact of road grade on carbon dioxide (CO2) emission of a passenger vehicle in real-world driving. Transp. Res. Part D Transp. Environ. 2014, 32, 160-170. [CrossRef]

43. Saerens, B. Optimal Control Based Eco-Driving. Ph.D. Thesis, Katholieke Universiteit Leuven, Leuven, Belgium, 2012.

44. Ahn, K. Microscopic Fuel Consumption and Emission Modeling. Master's Thesis, Virginia Polytechnic Institute and State University, Blacksburg, VA, USA, 1998.

45. Nam, E.K.; Giannelli, R. Fuel Consumption Modeling of Conventional and Advanced Technology Vehicles in the Physical Emission Rate Estimator (PERE), EPA-420-P-05-001; Assessment and Standards Division Office of Transportation and Air Quality, U.S. Environmental Protection Agency: Washington, DC, USA, 2005.

46. Stuhldreher, M.; Kargul, J.; Barba, D.; McDonald, J.; Bohac, S.; Dekraker, P.; Moskalik, A. Benchmarking a 2016 Honda Civic 1.5-Liter L15B7 Turbocharged Engine and Evaluating the Future Efficiency Potential of Turbocharged Engines. SAE Int. J. Engines 2018, 11, 1273-1305. [CrossRef]

47. Barth, M.; Scora, G.; Younglove, T. Modal Emissions Model for Heavy-Duty Diesel Vehicles. Transp. Res. Rec. 2004, 1880, 10-20. [CrossRef]

48. United States Environmental Protection Agency; National Highway Traffic Safety Administration. Greenhouse Gas Emissions and Fuel Efficiency Standards for Medium-And Heavy-Duty Engines and Vehicles-Phase 2; Office of Transportation and Air Quality, U.S. Environmental Protection Agency: Washington, DC, USA; National Highway Traffic Safety Administration, U.S. Department of Transportation: Washington, DC, USA, 2016. 
49. Frey, H.C.; Zhang, K.; Rouphail, N.M. Fuel Use and Emissions Comparisons for Alternative Routes, Time of Day, Road Grade, and Vehicles Based on In-Use Measurements. Environ. Sci. Technol. 2008, 42, 2483-2489. [CrossRef]

50. Levin, M.W.; Duell, M.; Waller, S.T. Effect of Road Grade on Networkwide Vehicle Energy Consumption and Ecorouting. Transp. Res. Rec. 2014, 2427, 26-33. [CrossRef]

51. United States Environmental Protection Agency. Development of Emission Rates for Light-Duty Vehicles in the Motor Vehicle Emissions Simulator (MOVES2010); United States Environmental Protection Agency: Washington, DC, USA, 2011.

52. Llopis-Castelló, D.; Pérez-Zuriaga, A.M.; Camacho-Torregrosa, F.J.; García, A. Impact of horizontal geometric design of two-lane rural roads on vehicle co 2 emissions. Transp. Res. Part D Transp. Environ. 2018, 59, 46-57. [CrossRef]

53. Wallace, J.M.; Hobbs, P.V. Atmospheric Science: An Introductory Survey, 2nd ed.; International Geophysics Series; Elsevier Academic Press: Cambridge, MA, USA, 2006; ISBN 978-0-12-732951-2.

54. Rexeis, M.; Hausberger, S.; Kühlwein, J.; Luz, R. Update of Emission Factors for EURO 5 and EURO 6 Vehicles for the HBEFA Version 3.2.; Institute for Internal Combustion Engines and Thermodynamics, Graz University of Technology: Graz, Austria, 2013; p. 74.

55. Sturm, P.J.; Hausberger, S. (Eds.) Energy and Fuel Consumption from Heavy Duty Vehicles. COST 346-Final Report; Institute for Internal Combustion Engines and Thermodynamics, Graz University of Technology: Graz, Austria, 2005; ISBN 978-3-902465-48-1.

56. Rexeis, M.; Quaritsch, M.; Hausberger, S.; Silberholz, G.; Kies, A.; Heinz, S.; Goschütz, M.; Vermeulen, R. VECTO Tool Development: Completion of Methodology to Simulate Heavy Duty Vehicles' Fuel Consumption and CO2 Emissions Upgrades to the Existing Version of VECTO and Completion of Certification Methodology to be Incorporated into a Commission Legislative Proposal; European Commission DG Clima; Graz University of Technology: Graz, Austria, 2017; p. 157.

57. Rakha, H.; Dion, F.; Sin, H.-G. Using Global Positioning System Data for Field Evaluation of Energy and Emission Impact of Traffic Flow Improvement Projects: Issues and Proposed Solutions. Transp. Res. Rec. 2001, 1768, 210-223. [CrossRef]

58. Liu, H.; Rodgers, M.O.; Guensler, R. Impact of road grade on vehicle speed-acceleration distribution, emissions and dispersion modeling on freeways. Transp. Res. Part D Transp. Environ. 2019, 69, 107-122. [CrossRef]

59. Mendes, M.M. Análise da Eficácia da Avaliação de Impactes da Rede Nacional de Auto-Estradas. Master's Thesis, Faculdade de Ciências e Tecnologia, Universidade Nova de Lisboa Lisbon, Portugal, 2012. 